\title{
A New Approach in Petrophysical Rock Typing
}

\author{
Abouzar Mirzaei-Paiaman ${ }^{1, *}$, Mehdi Ostadhassan², Reza Rezaee ${ }^{3}$, Hadi Saboorian-Jooybari ${ }^{4}$, \\ Zhangxin $\mathrm{Chen}^{4}$ \\ ${ }^{1}$ Department of Petroleum Engineering, National Iranian South Oil Company, Ahvaz, Iran \\ ${ }^{2}$ Petroleum Engineering Department, University of North Dakota, Grand Forks, ND 58201, USA \\ ${ }^{3}$ Department of Petroleum Engineering, Curtin University, Perth 6151, Australia \\ ${ }^{4}$ Department of Chemical and Petroleum Engineering, Schulich School of Engineering, University of Calgary, 2500 \\ University Drive NW, Calgary, AB, Canada T2N 1N4 \\ *Corresponding author: Mirzaei1986@gmail.com, Tel.:+989168014851
}

\section{Abstract}

Petrophysical rock typing in reservoir characterization is an important input for successful drilling, production, injection, reservoir studies and simulation. In this study petrophysical rock typing is divided into two major categories: 1) a petrophysical static rock type (PSRT): a collection of rocks having the same primary drainage capillary pressure curves or unique water saturation for a given height above the free water level, 2) a petrophysical dynamic rock type (PDRT): a set of rocks with a similar fluid flow behavior. It was shown that static and dynamic rock types do not necessarily overlap or share petrophysical properties, regardless of wettability. In addition, a new index is developed to define PDRTs via the Kozeny-Carman equation and Darcy's law. We also proposed a different index for delineation of PSRTs by combining the Young-Laplace capillary pressure expression and the Kozeny-Carman equation. These new indices were compared with the existing theoretical and empirical indices. Results showed that our indices are representatives of previously developed models which were also tested with mercury injection capillary pressure, water-oil primary drainage capillary pressure, and water-oil relative permeability data on core plugs from a highly heterogeneous carbonate reservoir in an Iranian oil field. This study enabled us to modify the conventional J-function to enhance its capability of normalizing capillary pressure data universally.

Key words: petrophyisics, rock typing, hydraulic flow unit, Kozeny-Carman, reservoir characterization

\section{Introduction}

Petrophysical rock typing has a wide variety of applications such as: drilling (e.g., prediction of high mud-loss intervals), production (e.g., potential production zones, locating perforations, diversion system design in acidizing, and prediction of high injectivity zones) (Roque et al., 
2017, Oliveira et al., 2016), reservoir studies (net-pay cut-off definition) (Kolodzie, 1980, Saboorian-Jooybari, 2017), representative sample selections for special core analysis (SCAL) tests (Siddiqui et al., 2006, Serag El Din et al., 2014, Mirzaei-Paiaman and Saboorian-Jooybari, 2016), permeability prediction in uncored intervals (Amaefule et al., 1993, Abbaszadeh et al., 1996, Davies and Vessell, 1996, Soto et al., 2001, Taslimi et al., 2008, Askari and Behrouz, 2011, Sokhal et al., 2016, Chen and Yao, 2017, Zhang et al. 2018, Wang et al. 2018), and defining saturation functions for static and dynamic reservoir models (Mirzaei-Paiaman et al., 2015, Askari and Behrouz, 2011). Generally the literature core-based petrophysical rock typing methods can be classified into three separate categories.

- Methods that utilize permeability, porosity and connate water saturation to some extent. Excluding the cut-off based methods (Rebelle, 2014) other ones are either empirical such as: Winland r35 (Kolodzie, 1980), Pittman equations (Pittman, 1992) and $r_{p 35}$ (Aguilera, 2002) or theoretical: FZI by Amaefule et al. (1993) and FZI* (FZI-Star) by MirzaeiPaiaman et al. (2015).

- Methods based on capillary pressure $\left(P_{c}\right)$ data such as a J-function, the empirical $P_{c}$ grouping technique, parameterization (Thomeer, 1960; Xu and Torres-Verdín, 2013; Lin et al., 2015) and measured r35 (Kolodzie, 1980).

- The spontaneous imbibition rate-driven method of FZI** or FZI-Double Star (MirzaeiPaiaman and Saboorian-Jooybari, 2016)

Among the above methods, the first category has caught major interest in industry and academia since its indices do not require prior knowledge of capillary pressure and/or relative permeability data. However, similar indices have been proposed that need SCAL-driven parameters (Nooruddin and Hossain, 2011; Izadi and Ghalambor, 2013), which makes their use much difficult. The theoretical and empirical indices are summarized in Table 1. The theoretical indices are mainly based on the generalized form of the Kozeny-Carman equation. Empirical methods have established a correlation between porosity, permeability, and a representative pore throat radius which is derived from mercury injection capillary pressure (MICP) data (Purcell, 1949). Such a specific pore throat radius has been introduced as an index for characterizing pore geometry in such a way that it dominates fluid flow. However, several shortcomings exist which led us to study them further and provide an enhanced definition (index): 
- In reality more than one pore throat size dominates fluid flow (usually a class of pore throat radii controls the flow).

- The presumed mathematical form of an equation that is used for a multiple regression analysis is subjective to the statistical analysis that has been used.

- Each publication has reported a different pore throat radius that controls the flow.

- In each study, for the same pore throat radius, different regression constants have been obtained.

Table 1 Empirical and theoretical petrophysical rock typing indices

\begin{tabular}{|c|c|c|c|c|}
\hline Author(s) & Type & Data/basis & Index & Comments \\
\hline $\begin{array}{c}\text { Winland } \\
\text { (Kolodzie, 1980) }\end{array}$ & Empirical & $\begin{array}{c}\text { A mixed collection of sandstones and } \\
\text { carbonates on Weyburn, Spindle and } \\
\text { Hidalgo fields in USA }\end{array}$ & $\begin{array}{l}\log \left(r_{35}\right) \\
=-0.996 \\
+0.588 \log (\mathrm{k}) \\
-0.864 \log (\phi)\end{array}$ & $\begin{array}{l}\text { Hereafter, } \mathrm{k} \text { is } \\
\text { permeability in } \\
\mathrm{mD}, \phi \text { is effective } \\
\text { porosity in fraction } \\
\text { and } \mathrm{r}_{\mathrm{i}} \text { is a } \\
\text { representative pore } \\
\text { throat radius } \\
\text { measured at } \\
\text { mercury saturation } \\
\text { of } i \% \text {. All indices } \\
\text { in this work are } \\
\text { expressed in } \\
\text { microns }\end{array}$ \\
\hline Kolodzie (1980) & Empirical & Spindle field shaly sandstone & $\begin{array}{l}\log \left(\mathrm{r}_{35}\right) \\
=-0.9008 \\
+0.5547 \log (\mathrm{k}) \\
-0.9033 \log (\phi)\end{array}$ & - \\
\hline Pittman (1992) & Empirical & $\begin{array}{l}202 \text { sandstones from } 14 \text { formations } \\
\text { from Ordovician to Tertiary }\end{array}$ & $\begin{array}{l}\log \left(\mathrm{r}_{20}\right) \\
=-0.388 \\
+0.519 \log (\mathrm{k}) \\
-0.303 \log (\phi) \\
\log \left(\mathrm{r}_{25}\right) \\
=-0.496 \\
+0.531 \log (\mathrm{k}) \\
-0.350 \log (\phi)\end{array}$ & - \\
\hline \multirow[t]{2}{*}{ Jaya et al. (2005) } & \multirow[t]{2}{*}{ Empirical } & \multirow[t]{2}{*}{$\begin{array}{l}273 \text { sandstones and } 42 \text { limestones from } \\
\text { Indonesian reservoirs }\end{array}$} & $\begin{array}{l}\log \left(r_{15}\right) \\
=0.293+0.694 \log (\mathrm{k}) \\
-3.604(\phi)\end{array}$ & For sandstones \\
\hline & & & $\begin{array}{l}\log \left(\mathrm{r}_{15}\right) \\
=0.549+0.883 \log (\mathrm{k}) \\
-4.399(\phi)\end{array}$ & For limestones \\
\hline \multirow[t]{2}{*}{ Ngo et al. (2015) } & \multirow[t]{2}{*}{ Empirical } & \multirow[t]{2}{*}{$\begin{array}{c}\text { Sandstone and carbonate rocks from } \\
\text { Cuu Long and Song Hong basins in } \\
\text { Vietnam }\end{array}$} & $\begin{array}{l}\log \left(\mathrm{r}_{55}\right) \\
=25.9814 \\
-2.6118 \log (\mathrm{k}) \\
+23.3716 \log (\phi)\end{array}$ & For sandstones \\
\hline & & & $\begin{array}{l}\log \left(\mathrm{r}_{20}\right) \\
=-1.4946 \\
+0.8155 \log (\mathrm{k}) \\
-1.5788 \log (\phi)\end{array}$ & For carbonates \\
\hline Aguilera (2002) & Empirical & $\begin{array}{l}\text { The empirical relationship between } \\
\qquad \text { MICP and } \frac{\mathrm{k}}{\phi} \text { developed by } \\
\text { Kwon and Pickett (1975) using } 2500 \\
\text { sandstone and carbonate samples from }\end{array}$ & $\begin{array}{l}\log \left(r_{35}\right) \\
=-0.4743 \\
+0.45 \log (\mathrm{k}) \\
-0.45 \log (\phi)\end{array}$ & - \\
\hline
\end{tabular}


30 different formations in North

America

\begin{tabular}{|c|c|c|c|c|}
\hline \multicolumn{5}{|c|}{ America } \\
\hline $\begin{array}{l}\text { Amaefule et al. } \\
\text { (1993) }\end{array}$ & Theoretical & $\begin{array}{c}\text { Generalized form of Kozeny-Carman } \\
\text { equation }\end{array}$ & $F Z I=\frac{0.0314 \sqrt{\frac{\mathrm{k}}{\phi}}}{\frac{\phi}{1-\phi}}$ & $\begin{array}{l}\text { FZI is flow zone } \\
\text { indicator. The } \\
\text { numerator was } \\
\text { named RQI or } \\
\text { reservoir quality } \\
\text { index }\end{array}$ \\
\hline $\begin{array}{l}\text { Jongkittinarukorn } \\
\text { and Tiab (1997) }\end{array}$ & Theoretical & $\begin{array}{l}\text { Application of several published shaly } \\
\text { sand models }\end{array}$ & $\mathrm{SZI}=\frac{0.0314 \sqrt{\frac{\mathrm{k}}{\phi}}}{\phi^{\mathrm{m}}}$ & $\begin{array}{l}\text { SZI is shale zone } \\
\text { indicator. In this } \\
\text { model, m depends } \\
\text { on the type of the } \\
\text { shale in the system } \\
(1.5 \text { for sand } \\
\text { reservoirs, } 2.5 \text { for } \\
\text { laminated shale and } \\
\text { structural shale, } \\
\text { and } 3.5 \text { for } \\
\text { dispersed shale })\end{array}$ \\
\hline $\begin{array}{l}\text { Nooruddin and } \\
\text { Hossain (2011) }\end{array}$ & Theoretical & $\begin{array}{l}\text { The generalized form of Kozeny- } \\
\text { Carman equation combined with two } \\
\text { other equations which one relates } \\
\text { tortuosity to formation resistivity } \\
\text { factor and porosity and the other one } \\
\text { relates formation resistivity factor to } \\
\text { lithology factor and cementation } \\
\text { exponent, m, (i.e. Archie's equation) }\end{array}$ & $\mathrm{FZIM}=\frac{0.0314 \sqrt{\frac{\mathrm{k}}{\phi}}}{\frac{\phi^{\mathrm{m}}}{1-\phi}}$ & $\begin{array}{l}\text { FZIM is a modified } \\
\text { FZI }\end{array}$ \\
\hline $\begin{array}{c}\text { Izadi and } \\
\text { Ghalambor (2013) }\end{array}$ & Theoretical & $\begin{array}{l}\text { The generalized form of Kozeny- } \\
\text { Carman equation and by taking into } \\
\text { account the irreducible (or connate) } \\
\text { water saturation, } \mathrm{S}_{\mathrm{wc}}\end{array}$ & $\begin{array}{l}\text { MFZI } \\
=\frac{0.0314 \sqrt{\frac{\mathrm{k}}{\phi}} \times \sqrt{1-\mathrm{S}_{\mathrm{wc}}}}{\frac{\phi}{1-\phi}\left(1-\mathrm{S}_{\mathrm{wc}}\right)^{2}}\end{array}$ & $\begin{array}{l}\text { MFZI is a modified } \\
\text { FZI. The numerator } \\
\text { was referred to } \\
\text { modified RQI }\end{array}$ \\
\hline $\begin{array}{l}\text { Ferreira et al. } \\
\quad(2015)\end{array}$ & Theoretical & $\begin{array}{l}\text { The base form of Kozeny-Carman } \\
\text { equation combined with Young- } \\
\text { Laplace capillary pressure equation }\end{array}$ & $\mathrm{RQI}^{*}=0.0314 \sqrt{\frac{\mathrm{k}}{\phi} \tau}$ & $\begin{array}{l}\text { RQI* is a modified } \\
\text { form of RQI and } \tau \\
\text { is hydraulic } \\
\text { tortuosity }\end{array}$ \\
\hline This study & Theoretical & $\begin{array}{l}\text { The base form of Kozeny-Carman } \\
\text { equation combined with Young-- } \\
\text { Laplace capillary pressure equation }\end{array}$ & PSRTI $=0.0314 \sqrt{\frac{\mathrm{k}}{\phi}} \mathrm{F}_{\mathrm{s}} \tau$ & $\begin{array}{l}\text { PSRTI is an index } \\
\text { for characterization } \\
\text { of PSRTs and } F_{S} \text { is } \\
\text { the shape factor }\end{array}$ \\
\hline & Theoretical & $\begin{array}{l}\text { The base form of Kozeny-Carman } \\
\text { equation and Darcy's law }\end{array}$ & $\mathrm{FZI}^{*}=0.0314 \sqrt{\frac{\mathrm{k}}{\phi}}$ & $\begin{array}{l}\text { FZI }^{*} \text { (FZI-Star) is a } \\
\text { modified FZI for } \\
\text { delineation of } \\
\text { PDRTs }\end{array}$ \\
\hline
\end{tabular}

We divide petrophysical rock typing into two categories of petrophysical static rock typing and petrophysical dynamic rock typing. Despite the differences that exist between petrophysical dynamic rock types (PDRTs) and petrophysical static rock types (PSRTs), these two have often been considered the same without a proper distinction. In many occasions the terms such as a petrophysical rock type, flow unit, and hydraulic flow unit (HFU) have interchangeably been used without any universal definition. Gholami and Mohaghegh (2009) presented an overview of different definitions that are used in the literature which are mostly qualitative and do not differentiate between PSRTs and PDRTs. 
We quantitatively define a PSRT as a collection of rocks with the same primary drainage capillary pressure curves or unique water saturation for a given height above the free water level (FWL). Identification of PSRTs is necessary in saturation-height calculations where the goal is to calculate water saturation at different heights above the FWL. Our definition naturally implies that any rock typing index that is developed from the drainage capillary pressure concept is only applicable to static rock typing. We further define a PDRT (or HFU) as a class of rocks with similar fluid flow behavior. Since the criterion "similar fluid flow behavior" is qualitative, it would not be incorrect to use Darcy's law to present an equivalent quantitative criterion. Delineation of PDRTs is critical in assigning saturation functions to simulation/dynamic models or whenever the purpose is to distinguish between flow capacity of rocks (e.g., perforation placement and mud-loss prediction).

Researchers have assumed that rocks with the same drainage capillary pressure curves have similar fluid flow behaviors (e.g. Saboorian-Jooybari et al. 2010, Izadi and Ghalambor, 2013, Ferreira et al., 2015). This means that, regardless of wettability, static and dynamic rock types are considered as one entity. However, it will be shown later, depending on the complexity of a porous medium, this definition will fail, especially in carbonate rocks. Therefore, rocks within one PSRT may represent separate PDRTs and vice versa. We emphasize that pore/network geometry is the main reason for different static and dynamic characteristics which must be taken into consideration for rock typing. Differentiation between static and dynamic rock types led us to develop separate indices to define PSRTs and PDRTs.

\section{Results}

\subsection{Index for dynamic rock typing}

We took two separate approaches to develop an index for characterization of PDRTs. We will first provide an overview of the basic and generalized forms of the Kozeny-Carman equation since the FZI methods by Amaefule et al. (1993), Nooruddin and Hossain (2011) and Izadi and Ghalambor (2013) were based on this equation. This is followed by derivation of a new index which is a general solution that integrates FZI, FZIM and MFZI. Our second approach utilizes Darcy's law for single and multiphase flow. Based on Darcy's law we quantitatively defined the criterion of "the same fluid flow behavior" to generate an index for petrophysical dynamic rock typing.

\subsubsection{The Kozeny-Carman Equation}


It is well understood that pore and network geometrical attributes control fluid flow. Any petrophysical rock typing index should contain such attributes including: a pore body size and shape, a pore throat size, an aspect ratio, a coordination number and pore arrangements. While these attributes can be determined for each sample individually for rock typing (Davies and Vessel, 1996), relationships between these micro-scale attributes and macro-scale easy-tomeasure parameters (i.e., porosity and permeability) can be made by theoretical studies. In this regard, the Kozeny-Carman equation is a relationship which connects micro-scale attributes with permeability and porosity. It is based on assuming a porous medium as a bundle of capillary tubes (Kozeny, 1927; Carman, 1937) and is derived by equating Hagen-Poissoulle's and Darcy's laws to establish a fundamental relationship between macroscopic properties of a porous medium and microscopic attributes (pore characteristics). We refer to this equation as "the base form of Kozeny-Carman":

$$
\mathrm{k}=\phi \frac{\mathrm{r}_{\mathrm{mh}}^{2}}{\mathrm{~F}_{\mathrm{s}} \tau}
$$

\section{Equation 1}

in which $r_{m h}$ is the effective or mean hydraulic unit radius (defined as the ratio of a crosssectional area to a wetted perimeter), $\tau$ is the hydraulic tortuosity (defined as the ratio of actual or microscopically-travelled length $\mathrm{L}_{\mathrm{a}}$ to the system straight or macroscopic length $\mathrm{L}$ ) (Shen and Chen, 2007; Yun et al. 2008; Cai et al., 2014; ), and $\mathrm{F}_{\mathrm{s}}$ is the shape factor introduced to account for non-circular capillary tubes $\left(F_{s}=2\right.$ for a circular tube). In this equation the group $\frac{r_{m h}^{2}}{F_{s} \tau}$ includes microscopic properties and describes geological characteristics of sedimentary rocks. The relationship between the mean hydraulic radius, $r_{m h}$, the surface area per unit grain volume, $\mathrm{S}_{\mathrm{gv}}$, and porosity can be expressed as (Kozeny, 1927; Carman, 1937):

$$
r_{m h}=\frac{1}{S_{g v}}\left(\frac{\phi}{1-\phi}\right)
$$

Equation 2

According to this equation a microscopic property $\left(\mathrm{r}_{\mathrm{mh}}\right)$ is decomposed into a microscopic property $\left(\mathrm{S}_{\mathrm{gv}}\right)$ and a macroscopic one $(\phi)$. Substituting Equation 2 in Equation 1 generates "the generalized form of Kozeny-Carman" as:

$$
k=\frac{\phi^{3}}{(1-\phi)^{2}}\left(\frac{1}{F_{s} \tau S_{g v}^{2}}\right)
$$




\section{Equation 3}

In the resulting equation the group $\mathrm{F}_{\mathrm{s}} \tau \mathrm{S}_{\mathrm{gv}}^{2}$ also contains microscopic properties of rocks. Amaefule et al. (1993) rearranged this equation as:

$$
\log (\mathrm{RQI})=\log \left(\frac{\phi}{1-\phi}\right)+\log (\mathrm{FZI})
$$

Equation 4

where RQI and FZI, both in $\mu \mathrm{m}$, are defined as:

$$
\mathrm{RQI}=0.0314 \sqrt{\frac{\mathrm{k}}{\phi}}
$$

Equation 5

$$
\mathrm{FZI}=\frac{\mathrm{RQI}}{\frac{\phi}{1-\phi}}=\frac{1}{\sqrt{\mathrm{F}_{\mathrm{s}} \tau} \mathrm{S}_{\mathrm{gv}}}
$$

Equation 6

Since the aforementioned microscopic properties are initially unknown for each rock, Amaefule

et al. (1993) presented a single variable called FZI. However, the problem raises if $F Z I=\frac{1}{\sqrt{\mathrm{F}_{\mathrm{s}} \tau} \mathrm{S}_{\mathrm{gv}}}$ can perfectly characterize different zones in terms of their flow characteristics. If it is assumed that a porous medium contains layers of uniform spheres, the surface area per unit grain volume, $\mathrm{S}_{\mathrm{gv}}$, can be written as:

$$
\mathrm{S}_{\mathrm{gv}}=\frac{6}{\mathrm{D}_{\mathrm{p}}}
$$

Equation 7

where $D_{p}$ is the particle or grain diameter. Inserting Equation 7 in Equation 6 gives:

$$
\mathrm{FZI}=\frac{\mathrm{RQI}}{\frac{\phi}{1-\phi}}=\frac{\mathrm{D}_{\mathrm{p}}}{6 \sqrt{\mathrm{F}_{\mathrm{s}} \tau}}
$$

Equation 8

In terms of microstructure characteristics of porous media this equation shows that FZI is a function of a grain diameter, a shape factor, and hydraulic tortuosity. The quantity of direct interest in the fluid flow through porous media is not a property of the solid component (e.g., a grain diameter) but rather a property of pores (e.g., a pore size). In fact, we are interested to know the corresponding pore space rather than the matrix volume. A closer look at Equation 1 
reveals that $\frac{r_{m h}^{2}}{F_{s} \tau}$ is the correct bundle of microstructural attributes of sedimentary rocks. In addition to the shape factor $\left(F_{s}\right)$ and hydraulic tortuosity $(\tau), \frac{r_{m h}^{2}}{F_{s} \tau}$ contains $r_{m h}$ which is the quantity of direct interest in the fluid flow through porous media. Hence, if fluid properties remain unchanged, Hagen-Poissoulle's equation results in $\frac{r_{m h}^{2}}{F_{s} \tau}$ to vary between the $\frac{v}{d p / d x}$ values of different rock types ( $\mathrm{v}$ is the fluid velocity or interstitial velocity and $\frac{\mathrm{dp}}{\mathrm{dx}}$ is the pressure change per unit length of a porous medium). Consequently, the term $\frac{\mathrm{v}}{\mathrm{dp} / \mathrm{dx}}$ can be used to characterize fluid flow behavior of a rock. Previously, we used $\frac{r_{m h}^{2}}{F_{s} \tau}$ as the correct flow zone indicator and to create Equation 1 as below (Mirzaei-Paiaman et al., 2015):

$$
\text { FZI }^{*}=0.0314 \sqrt{\frac{\mathrm{k}}{\phi}}=\frac{\mathrm{r}_{\mathrm{mh}}}{\sqrt{\mathrm{F}_{\mathrm{s}} \tau}}
$$

Equation 9

In this equation FZI* (FZI-Star) in $\mu \mathrm{m}$ is the correct flow zone indicator.Thus, rocks with similar FZI* show similar fluid flow behavior as a single PDRT. The numerical value of FZI* can be calculated using Equation 9 for each rock with known measured porosity and permeability values. Furthermore, graphically a plot of $0.0314 \sqrt{\mathrm{k}}$ versus $\sqrt{\phi}$ on a $\log$-log scale becomes a straight line with the slope of unity for rock samples within one PDRT or HFU. The intercept of a unit-slope straight line with the coordinate $\phi=1$ is the FZI*. Likewise, samples with different FZI* lay on a series of parallel unit-slope straight lines. Therefore, to predict permeability in uncored sections, the following equation can be used:

$$
\mathrm{k}=1014 \phi\left(\mathrm{FZI}^{*}\right)^{2}
$$

\section{Equation 10}

$\mathrm{k} / \phi$ for reservoir rock characterization, or as Amaefule et al. (1993) called it RQI, has been used in the petroleum industry for decades. Besides it has been used in the Leverett J-function for normalizing capillary pressure data. This term is also known as a process or delivery speed for reservoir zonation (Chopra, 1988; Chopra et al., 1989; Aguilera, 2004). Xu and Torres-Verdín (2012) used RQI to classify hydraulic rock types based on core measurements. The identified hydraulic rock types were then used to rank saturation-dependent capillary pressure and relative permeability data. Despite the different names for this group, this term only indicates the flow 
deliverability potential of a rock. Thus, we use the term FZI* to directly define the ability of this index to characterize the flow deliverability of the zone of interest.

\subsubsection{Darcy's law for single and multi-phase flow}

One-dimensional flow of a single phase fluid in a homogeneous rock can be written by Darcy's law as:

$$
\mathrm{u}=-\frac{\mathrm{k}}{\mu} \frac{\mathrm{dp}}{\mathrm{dx}}
$$

Equation 11

where $\mathrm{u}$ is a velocity and $\mu$ is the fluid viscosity. In this equation, $\mathrm{u}$ is often referred to as the Darcy velocity. The fluid velocity or interstitial velocity $\mathrm{v}$ is related to the Darcy velocity through porosity to show the fraction of the total rock volume available for flow:

$$
\mathrm{u}=\mathrm{v} \phi
$$

Equation 12

Inserting Equation 12 in Equation 11 results in:

$$
\frac{\mathrm{v}}{\mathrm{dp} / \mathrm{dx}}=-\frac{\mathrm{k}}{\phi} \frac{1}{\mu}
$$

Equation 13

As stated before, in this equation the term $\frac{\mathrm{v}}{\mathrm{dp} / \mathrm{dx}}$ can be used to characterize fluid flow behavior of rocks. Thus, systems with similar fluid flow characteristics are expected to have identical $\frac{\mathrm{v}}{\mathrm{dp} / \mathrm{dx}}$. In the above equation $\frac{\mathrm{k}}{\phi}$ and $\mu$ account for rock and fluid properties, respectively. If $\mu$ remains constant then $\frac{\mathrm{k}}{\phi}$ is the only parameter that controls fluid flow. Accordingly, for single phase flow, Darcy's law shows that FZI* becomes the most appropriate petrophysical rock typing index that characterizes flow zones. In multiphase flow, Darcy's law for water, oil and gas can be expressed as:

$$
\frac{\mathrm{v}_{\mathrm{w}}}{\mathrm{dp}_{\mathrm{w}} / \mathrm{dx}}=-\frac{\mathrm{k}_{\mathrm{ew}}}{\phi} \frac{1}{\mu_{\mathrm{w}}}=-\frac{\mathrm{kk}_{\mathrm{rw}}}{\phi} \frac{1}{\mu_{\mathrm{w}}}
$$

Equation 14

$$
\frac{\mathrm{v}_{\mathrm{o}}}{\mathrm{dp}_{\mathrm{o}} / \mathrm{dx}}=-\frac{\mathrm{k}_{\mathrm{eo}}}{\phi} \frac{1}{\mu_{\mathrm{o}}}=-\frac{\mathrm{kk}_{\mathrm{ro}}}{\phi} \frac{1}{\mu_{\mathrm{o}}}
$$

Equation 15 


$$
\frac{\mathrm{v}_{\mathrm{g}}}{\mathrm{dp}_{\mathrm{g}} / \mathrm{dx}}=-\frac{\mathrm{k}_{\mathrm{eg}}}{\phi} \frac{1}{\mu_{\mathrm{g}}}=-\frac{\mathrm{kk}_{\mathrm{rg}}}{\phi} \frac{1}{\mu_{\mathrm{g}}}
$$

\section{Equation 16}

where subscripts $\mathrm{w}$, o and $\mathrm{g}$ denote water, oil and gas, respectively, $\mathrm{k}_{\mathrm{e}}$ is effective permeability, and $k_{r}$ is relative permeability. Thus, systems with similar multiphase flow characteristics are expected to have equal $\frac{\mathrm{v}_{\mathrm{w}}}{\mathrm{dp}_{\mathrm{w}} / \mathrm{dx}}, \frac{\mathrm{v}_{\mathrm{o}}}{\mathrm{dp}_{\mathrm{o}} / \mathrm{dx}}$ and $\frac{\mathrm{v}_{\mathrm{g}}}{\mathrm{dp}_{\mathrm{g}} / \mathrm{dx}}$. If fluid properties remain constant then $\frac{\mathrm{k}_{\mathrm{e}}}{\phi}$ or $\frac{\mathrm{kk}_{\mathrm{r}}}{\phi}$ is the only parameter that controls fluid flow. This means rocks with similar flow characteristics can show similar $\frac{k k_{r}}{\phi}$. This becomes very important in reservoir characterization when analyzing SCAL data to identify different PDRTs. Although $k_{r}$ is a function of saturation, FZI* can be considered as the petrophysical rock typing index that characterizes flow zones in multiphase flow. Later in this manuscript we will use field data of a highly heterogeneous carbonate formation in the southwestern part of Iran to show that PDRTs can be identified by FZI* with distinct $\frac{k k_{r}}{\phi}$ versus saturation curves.

\subsection{Index for static rock typing}

A PSRT is a class of rocks with the same primary drainage capillary pressure curves having unique water saturation for a given height above FWL. To define a PSRT via capillary pressure, Young-Laplace is written as:

$$
\mathrm{P}_{\mathrm{c}}=\frac{\gamma \cos (\theta)}{\mathrm{r}_{\mathrm{mh}}}
$$

\section{Equation 17}

in which $\mathrm{P}_{c}$ is the capillary pressure, $\gamma$ is the interfacial tension between wetting and nonwetting fluids, $\theta$ is the contact angle and $r_{m h}$ is the mean hydraulic radius. Inputting Equation 1 in Equation 17 yields:

$$
\mathrm{P}_{\mathrm{c}}=\frac{\gamma \cos (\theta)}{\sqrt{\frac{\mathrm{k}}{\phi} \mathrm{F}_{\mathrm{s}} \tau}}
$$

\section{Equation 18}

According to this equation, if fluid properties and rock-fluid interaction properties are kept constant, rocks with similar $\sqrt{\frac{k}{\phi} F_{s} \tau}$ will have similar $P_{c}$ curves and constitute a PSRT. Therefore, 
the correct PSRT indicator (PSRTI) in $\mu \mathrm{m}$ is $0.0314 \sqrt{\frac{\mathrm{k}}{\phi} \mathrm{F}_{\mathrm{s}} \tau}$ or $\mathrm{FZI}^{*} \sqrt{\mathrm{F}_{\mathrm{s}} \tau}$. In the petroleum industry, if $\mathrm{F}_{\mathrm{s}} \tau$ is not measured for each rock individually, then $\mathrm{FZI}^{*}$ is the only parameter that may be used. Though, its accuracy depends on complexity of pore geometry. If all rocks have a similar $\mathrm{F}_{\mathrm{s}} \tau$ value then $\mathrm{FZI}^{*}$ would be sufficient for static rock typing. However, the assumption of the same $\mathrm{F}_{\mathrm{s}} \tau$ may not happen always, and thus $\mathrm{FZI}^{*}$ should be used with caution. The term $\mathrm{F}_{\mathrm{s}} \tau$ that appears in PSRTI can be determined from MICP experiments (Purcell, 1949). Then a correlation can be established to predict its value from well log data if lab measurements are not available. Geostatistical techniques can be used to calculate a saturation-height to determine initial water saturation in geologic models.

\subsection{Relationship between PSRT and PDRT}

In order to define the relationship between PSRT and PDRT, it is assumed that all fluid and rockfluid interaction properties are kept constant. In such a case, a porous medium is considered as a bundle of capillary tubes which enables us to use the Kozeny-Carman equation. We consider two different scenarios: 1) two porous media with similar capillary pressure curves (i.e., forming one PSRT) but exhibiting different flow characteristics (i.e., falling in different PDRTs), and 2) two porous media with different capillary pressure curves (i.e., not in one PSRT) but exhibiting similar flow characteristics (i.e., in one PDRT). The first case scenario is shown in Figure 1 where both media have a length $L$. Medium A is comprised of two circular straight capillary tubes (i.e., $\tau=1$ ) of radius $r$ while medium $\mathrm{B}$ is characterized by a single circular tortuous capillary tube with radius $r$ and $\tau=2$. Because of a similar hydraulic radius and porosity, these two porous media will have similar capillary pressure-liquid saturation curves and constitute a single PSRT. However, the Kozeny-Carman equation yields $\frac{k}{\phi}=\frac{r^{2}}{4}$ for system A and $\frac{k}{\phi}=\frac{r^{2}}{16}$ for system B reflecting different PDRTs. In the second scenario which is depicted in Figure 2, both media have length $L$. Porous medium A is made up of four circular tortuous capillary tubes (i.e., $\tau=2$ ) of radius $r$ while porous medium B is characterized by a single circular tortuous capillary tube with radius $2 r$ and $\tau=2$. Because of different hydraulic radii, these two porous media exhibit different capillary pressure-liquid saturation curves and constitute two different PSRTs. The Kozeny-Carman equation yields $\frac{k}{\phi}=\frac{r^{2}}{4}$ for both systems making them one PDRT. 
A

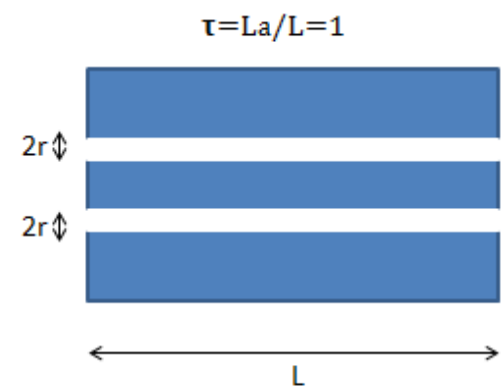

B

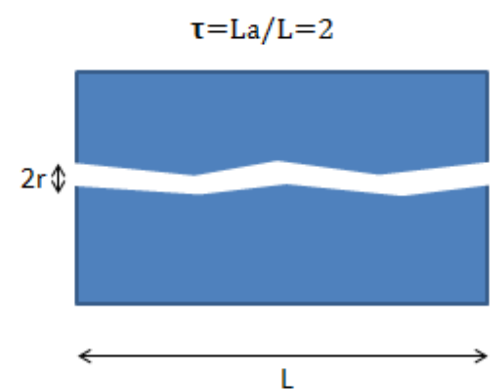

Figure 1 Schematic representation of two porous media with similar capillary pressure curves (i.e. forming one PSRT) but exhibiting different flow characteristics (i.e. forming different PDRTs)

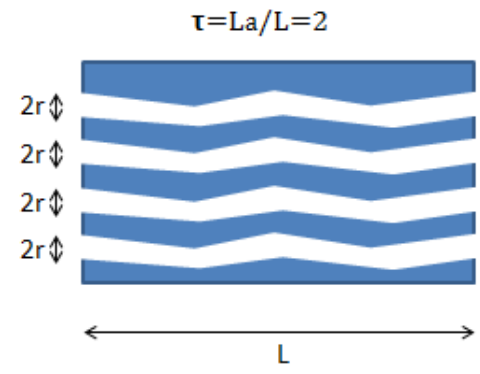

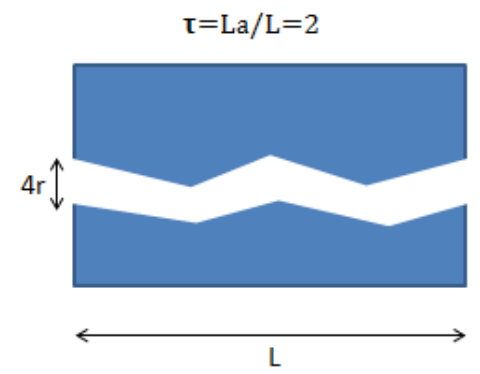

Figure 2 Schematic representation of two porous media with different capillary pressure curves (i.e. not in one PSRT) but exhibiting similar flow characteristics (i.e. forming one PDRT)

\subsection{Relationship between empirical indices and FZI* and PSRTI}

The presented index in this study for identification of PSRT $\left(P S R T I=0.0314 \sqrt{\frac{\mathrm{k}}{\phi} \mathrm{F}_{\mathrm{s}} \tau}\right)$ can be written as:

$$
\log (\mathrm{PSRTI})=\log \left(0.0314 \sqrt{\mathrm{F}_{\mathrm{s}} \tau}\right)+0.5 \log (\mathrm{k})-0.5 \log (\phi)
$$

Equation 19

Similarly, the FZI* for study of PDRTs can be expressed as:

$$
\log \left(\mathrm{FZI}^{*}\right)=-1.50307+0.5 \log (\mathrm{k})-0.5 \log (\phi)
$$

Equation 20

Interestingly the mathematical forms of these two equations reveal that previous empirical indices retain similar forms. Therefore, if the general index for petrophysical rock typing has the following form, the literature empirical indices will optimize the associated constants $C_{1}, C_{2}$ and 
$C_{3}$ as shown in Table 2 with the exception of Jaya et al. (2005) where a different mathematical form was used for multiple regression analysis:

$$
\log (\text { index })=C_{1}+C_{2} \log (\mathrm{k})+C_{3} \log (\phi)
$$

Equation 21

It is worth mentioning that $C_{1}$ in the case of PSRTI varies for each rock sample.

Table 2 Different indices and their constants

\begin{tabular}{|c|c|c|c|c|}
\hline Author(s) & Index & $C_{1}$ & $C_{2}$ & $C_{3}$ \\
\hline $\begin{array}{c}\text { Winland (Kolodzie, } \\
1980)\end{array}$ & $r_{35}$ & -0.996 & 0.588 & -0.864 \\
\hline Kolodzie (1980) & $r_{35}$ & -0.9008 & 0.5547 & -0.9033 \\
\hline \multirow{2}{*}{ Pittman (1992) } & $\mathrm{r}_{20}$ & -0.388 & 0.519 & -0.303 \\
\cline { 2 - 5 } & $\mathrm{r}_{25}$ & -0.496 & 0.531 & -0.350 \\
\hline \multirow{2}{*}{ Ngo et al. (2015) } & $\mathrm{r}_{55}$ & 25.9814 & -2.6118 & 23.3716 \\
\cline { 2 - 5 } & $\mathrm{r}_{20}$ & -1.4946 & 0.8155 & -1.5788 \\
\hline Aguilera (2002) & $\mathrm{r}_{35}$ & -0.4743 & 0.45 & -0.45 \\
\hline \multirow{2}{*}{ This study } & PSRTI & $\log \left(0.0314 \sqrt{\mathrm{F}_{\mathrm{s}}} \mathrm{r}\right)$ & 0.5 & -0.5 \\
\cline { 2 - 5 } & FZI* & -1.50307 & 0.5 & -0.5 \\
\hline
\end{tabular}

\subsection{Case Study}

To check and compare different petrophysical rock typing indices, a carbonate oil reservoir in the southwestern Iran has been used. The Albian-Campanian Bangestan group, containing Ilam and Sarvak Formations, in Ahvaz field (Figure 4) is one of the most giant Middle Eastern hydrocarbon reservoirs. In this study core-plug data of five wells were provided by National Iranian South Oil Company (NISOC). Thin section petrographic studies depict that the lithofacies can be classified using Dunham's (1962) classification, as majorly packstone and grainstone, occasionally wackstone and rarely mudstone (Figure 3). Diagenetic processes in this reservoir have significantly altered the original rock fabric through dissolution (creating vuggy, moldic, channel and micro-fracture secondary porosity types), compaction (dissolution seams and stylolites), dolomitization and cementation. 

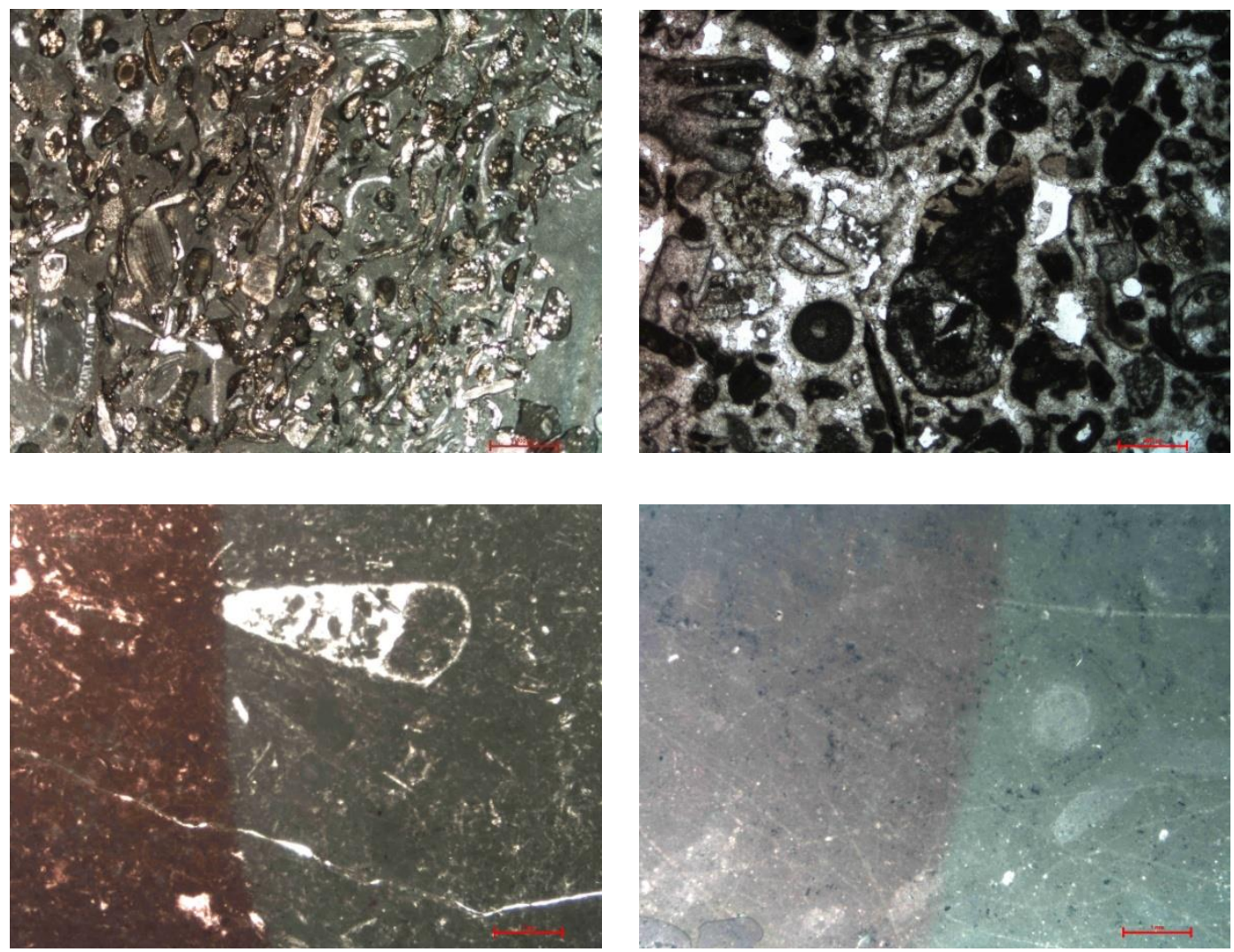

Figure 3 Different lithofacies observed in the studied wells; packstone (top left), grainstone (top right), wackstone (bottom left) and mudstone (bottom right)

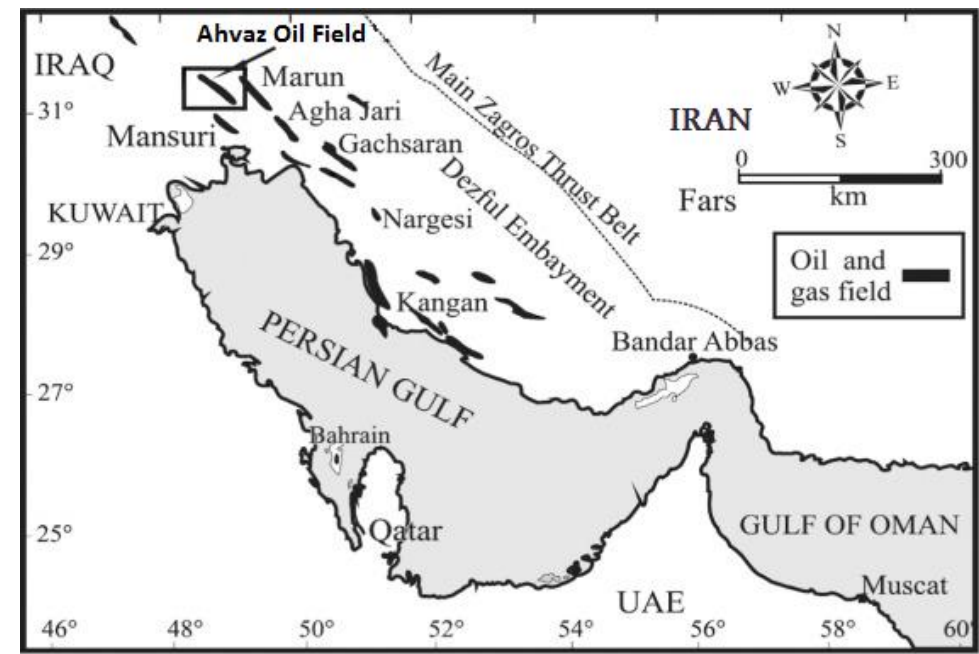

Figure 4 Regional location map of Ahvaz oil field (Tabatabaei et al., 2015)

Due to the complex nature of the pore system, when reservoir heterogeneity increases, characterization of PSRTs and PDRTs will become more challenging. In this study, we used a porosity-permeability cross-plot from RCAL data to show the level of complexity and heterogeneity. For horizontally and vertically retrieved core-plugs at a given value of porosity, 
permeability varies over several orders of magnitude proving the highly heterogeneous characteristics of the reservoir understudy (Figure 5).

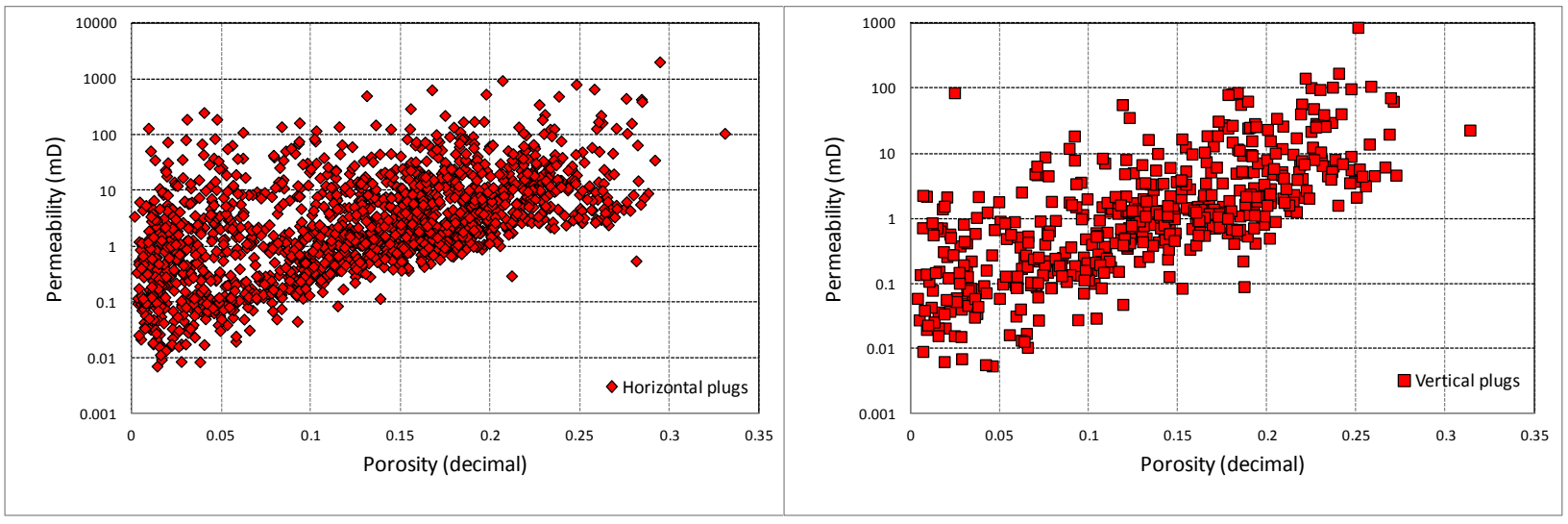

Figure 5 Porosity-permeability cross-plot from RCAL study for horizontal (left) and vertical (right) core-plugs

A collection of various data including: MICP, water-oil primary drainage capillary pressure, and water-oil relative permeability, strongly dependent on pore geometry and microstructural characteristics, are integrated and analyzed to compare different petrophysical rock typing indices. Rock samples within a PSRT must show similar primary drainage capillary pressure curves (either MICP or water-oil) forming an individual cluster. Since more than one PSRT is expected, several clusters representing several PSRTs appear. Furthermore, rocks within one PDRT must show similar fluid flow behavior (i.e., dynamic state). Therefore, fluid flow data are needed to confirm petrophysical dynamic rock typing indices. Previously, we (Mirzaei-Paiaman and Saboorian-Jooybari, 2016) acquired the rate of spontaneous imbibition recovery for validating the FZI** (FZI-Double Star) index whereas in this study water and oil relative permeability data are used. Totally, 34 MICP, 66 water-oil primary capillary pressure, and 62 water-oil relative permeability tests were conducted on horizontal and vertical plugs with porosities and permeabilities varying from 0.05 to 0.26 and 0.14 to $122.67 \mathrm{mD}$, respectively.

\subsubsection{PSRTs using capillary pressure data}

\subsubsection{MICP data}

We evaluate the ability of FZI*, FZI and Winland r35 in identification of PSRTs. PSRTI was excluded since it requires the knowledge of rock microstructures (i.e., $F_{s} \tau$ ). Such microstructural data can be determined for each sample by integration of MICP curves as described by Purcell (1949). However when MICP curves are utilized for verifying the performance of different indices, $F_{s} \tau$ should be determined from other independent sources of data such as petrographic 
images (Davies and Vessel, 1996) which were not available. FZI and Winland r35 are considered as representatives of theoretical and empirical indices, respectively. These two have widely been used and do not require complex input parameters. MICP data with other rock properties (i.e., porosity and permeability) are available in Supplementary Material. For each sample the numeral values of FZI*, FZI and Winland r35 are calculated using the relevant equations described previously. Ideally there should exist one single value for each PSRT/PDRT. However, a distribution of values around the mean is calculated (Chen and Zhou, 2017). In such cases, when more than one PSRT/PDRT exists, the overall distribution of that index is a superposition of the individual distributions around their mean value. Thus, identification of each mean index needs data deconvolution of the overall index distribution. Data clustering can be performed using statistical techniques such as discrete rock typing (DRT), histograms, probability plots and GHE or Global Hydraulic Element (Abbaszadeh et. 1996; Corbett and Potter, 2004; Mirzaei-Paiaman et al., 2015). In this study, we used the DRT technique due to its simplicity. In the following DRT, $C_{4}$ is a constant and can be adjusted for each index to make the outcome starting from 1 (i.e., PSRT1). Our data required $C_{4}$ values of 2.7 for FZI* and 1.7 for FZI and Winland r35.

$$
\text { DRT no. }=\operatorname{ROUND}\left(\mathrm{LOG}(\text { index })+C_{4} ; 0\right)
$$

\section{Equation 22}

The calculated values for each index and the corresponding DRT numbers are also included in Supplementary Material. In the following analysis, rock types 1, 2 and 3 are color coded as red, orange and green, respectively. It should be mentioned that DRT numbering is just to separate the overall index distribution into constituent clusters.

MICP curves are generally composed of three distinct sections, each corresponding to a certain saturation interval. Section one is at low mercury saturations where the capillary pressure curve usually shows a steep increase. This part corresponds to the largest pore throat sizes due to the irregularities on the sample surface. Thus, this data may not be truly representative of the rock microstructures. Section two initiates with a threshold pressure $P_{t h}$, and covers a wide range of saturations while a change in capillary pressure is dependent upon a pore throat size distribution. This saturation range starts with the mercury intruding the pores and thus it can represent true pore geometry of the rock that is involved in fluid flow. Finally, when capillary pressure reaches very large values, the finest pore throats that do not contribute to the flow start to get filled up 
with mercury. The latest data also do not truly represent the effective microstructures of the sample. In this study, we have focused on section two of the capillary pressure curve.

Figure 6 displays the MICP curves and the resulting PSRTs by FZI, FZI* and Winland r35 methods. FZI* and Winland r35 predict three PSRTs while FZI predicts only two clusters. According to the Young-Laplace capillary pressure equation, capillary pressure is proportional to the reciprocal of $r_{m h}$ meaning that as $r_{m h}$ decreases, $P_{c}$ increases. Therefore, MICP curves of PSRTs with smaller DRT numbers should place above the MICP curves of PSRTs with larger DRT numbers. With a comparison of three plots in Figure 6, a better performance of FZI* in identification of PSRTs is concluded. PSRT with DRT=1 (red color) situates above, PSRT with $\mathrm{DRT}=3$ (green color) is in the lowest region of the plot and PSRT with DRT=2 (orange) appears in-between. FZI fails completely to identify PSRTs and the predicted PSRTs by this method do not form distinct clusters. Winland r35 results in a better data clustering than FZI, and worse than FZI*. It was found that FZI* has a better performance than other techniques; however, its ability to identify PSRTs was not as expected. Also, the FZI* plot exhibits some MICP curves that do not follow the anticipated trends which means some rocks identified by FZI* as PSRT1 will fall in the PSRT2 cluster. The reasons for such behavior will be discussed later in text. 

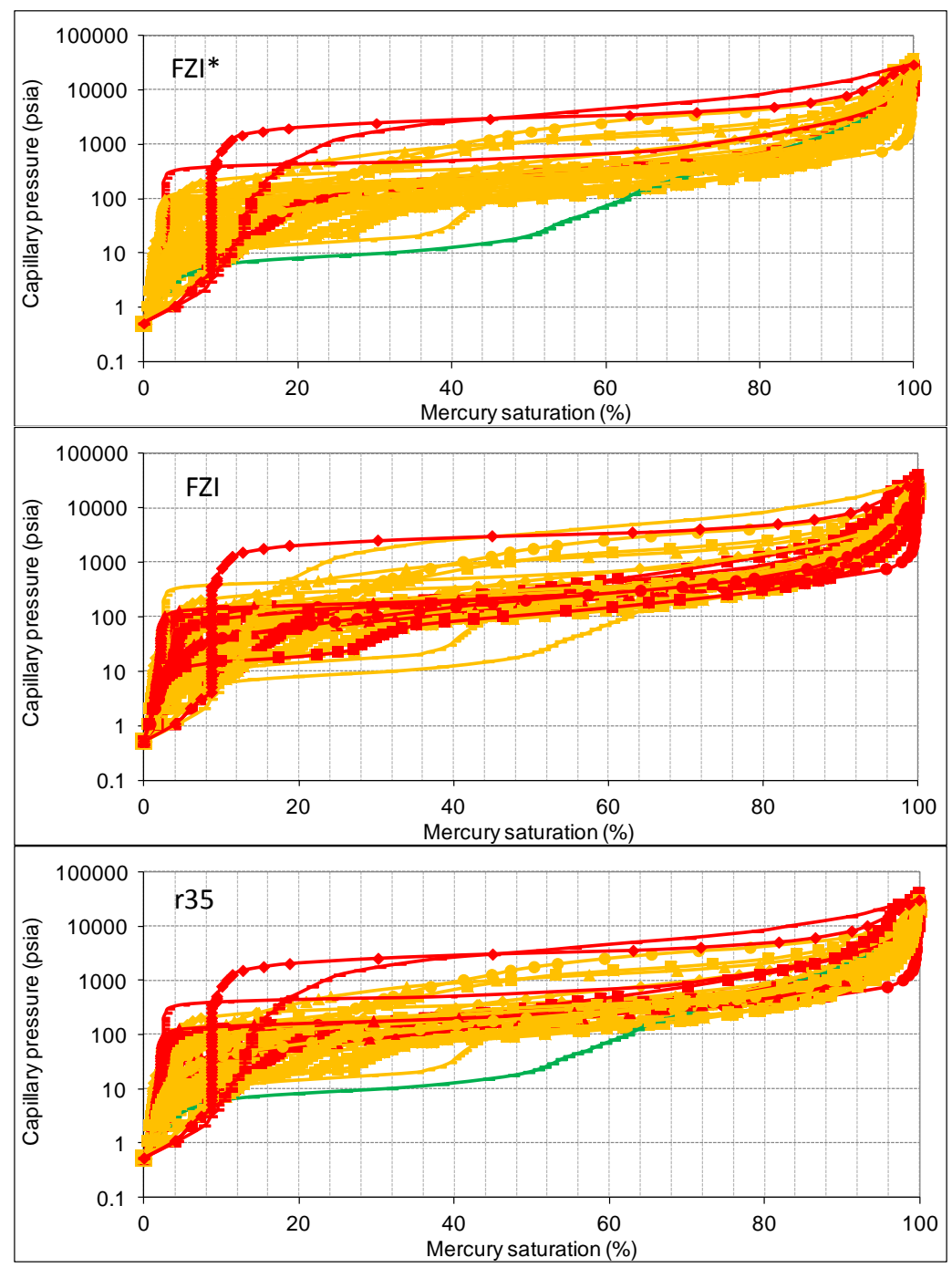

Figure 6 The MICP curves and the resulting PSRTs by FZI* (top), FZI (middle) and Winland r35 (bottom)

Assuming that a porous medium is a bundle of circular tubes, MICP curves can be converted to incremental mercury saturation vs. a pore throat radius through the Young-Laplace capillary pressure equation. The resulting curves are shown in Figure 7 where PSRTs that are predicted by each index are also shown. In this figure the data of aforementioned sections two and three of MICP curves are only used. Ideally each PSRT must have similar pore throat size distributions; therefore, in this figure it is expected to observe distinct clusters. Comparison of different plots in this figure reveals that FZI* has a significantly better performance than FZI and Winland r35. Winland r35, despite inferior performance than FZI*, is more accurate than FZI in identifying PSRTs. 

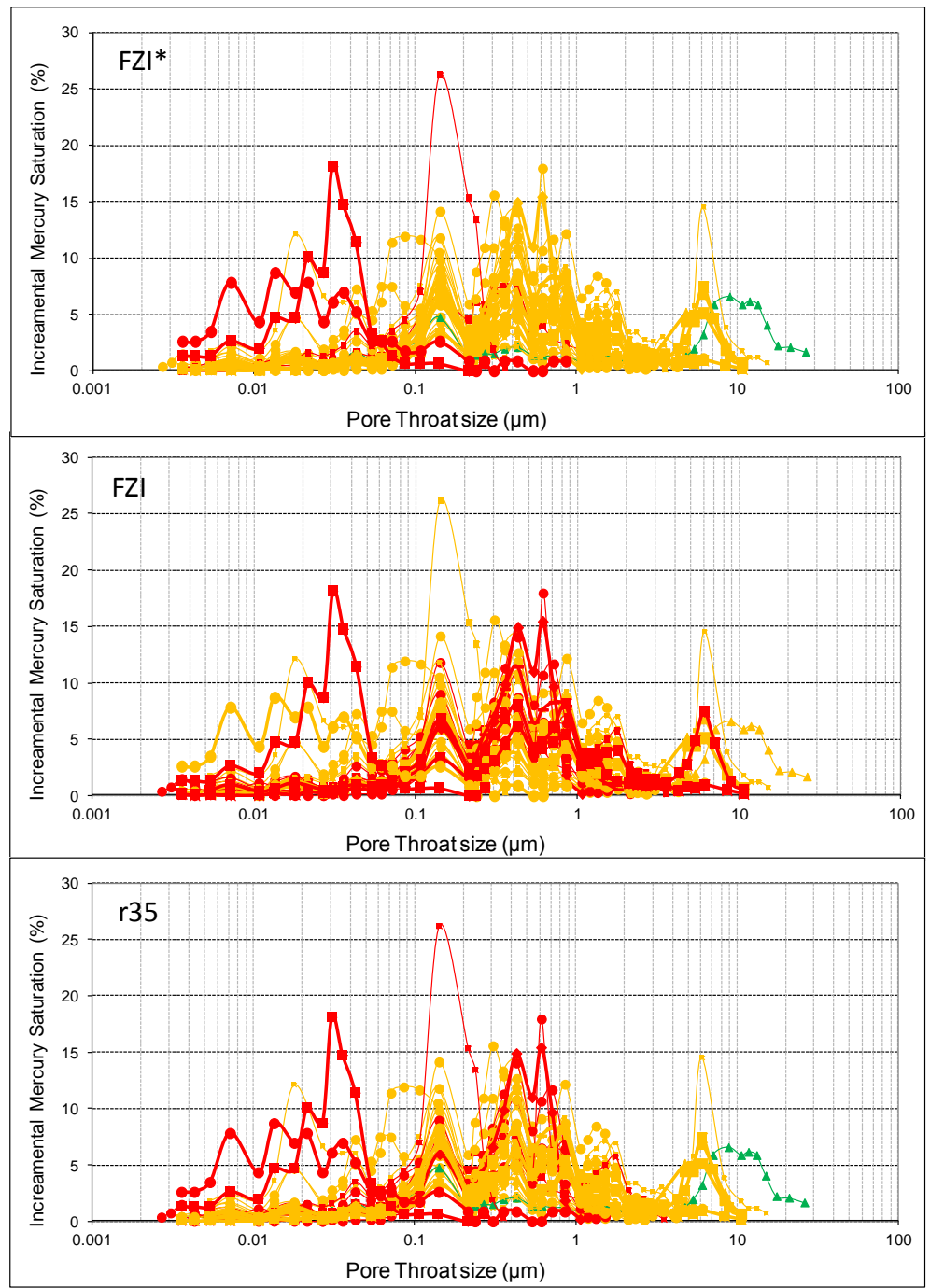

Figure 7 The incremental mercury saturation vs. pore throat radius curves and the resulting PSRTs by FZI* (top), FZI (middle) and Winland r35 (bottom)

To investigate the relationship between different indices and the threshold pressure $\left(\mathrm{P}_{\mathrm{th}}\right)$ this parameter was obtained for each MICP curve as well. Donaldson (1991) proposed extrapolation of a capillary pressure curve to mercury saturation of $100 \%$ to determine the threshold pressure. Katz and Thompson (1986) took the inflection point on a MICP curve to represent the threshold pressure. In this study on the log-log plot of capillary pressure vs. saturation, the intersection of two fitted straight lines through the data points on two separate sides of the inflection point is considered as the threshold pressure. An example is shown in Figure 8 and the calculated threshold pressures are listed in Supplementary Material.

Figure 9 explains the relationship between different indices and the threshold pressure. As $P_{t h}$ increases the indices should decrease. Here we compare the indices from two separate 
perspectives, 1) in terms of the correlation coefficient FZI* has the highest $R^{2}=0.44$, Winland r35 is the next $\left(R^{2}=0.30\right)$ and FZI with $R^{2}=0.11$ stands the last, 2$)$ with respect to the $P_{t h}$ range for each PSRT, where the PSRTs identified by FZI* have distinct ranges and the PSRTs by FZI have almost similar ranges. Considering Winland r35, PSRT1 and PSRT2 express significant overlapping.

To further investigate the relationship between different indices and other major parameters of capillary pressure curves, we fit Thomeer's (1960) model to our MICP data. Although the quality of match cases was not acceptable overall, we tried to obtain the Thomeer geometrical factor from the matches. We were not able to establish any meaningful relationship between the indices and geometrical factor which might be due to the complex pore structure of the samples caused by severe digenetic processes.

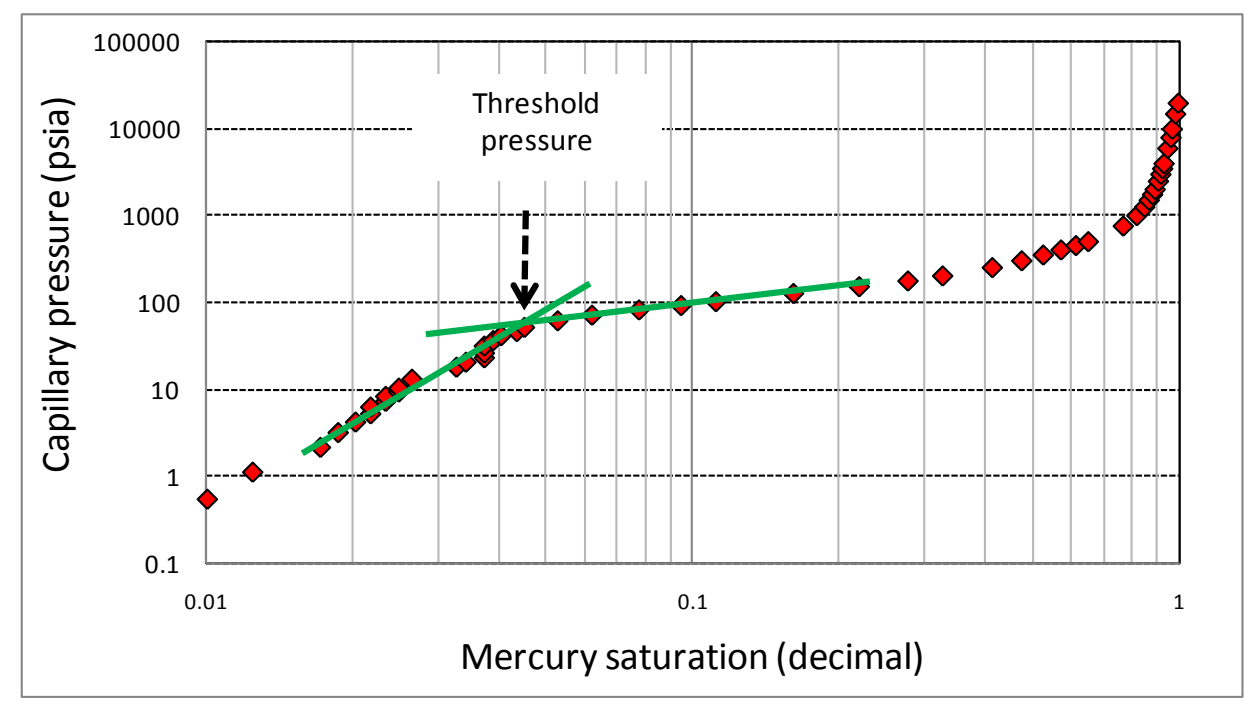

Figure 8 Graphical representation of the procedure used to determine threshold pressure from MICP data 


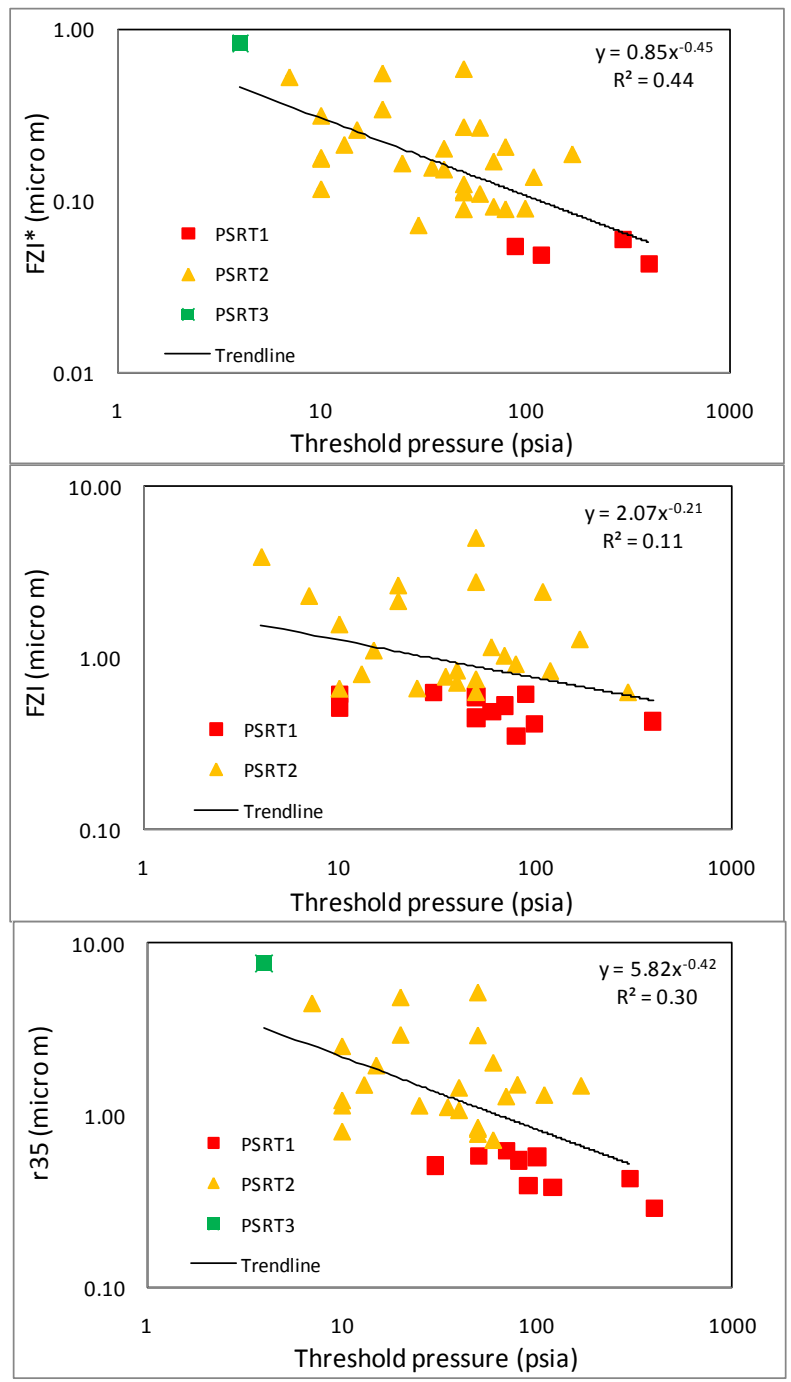

Figure 9 The relationship between different indices and the threshold pressure

We also obtained the experimentally measured r35 for each sample (see Supplementary Material). Various indices are also plotted vs. measured r35s and shown in Figure 10. Considering Winland $\mathrm{r} 35$, the correlation coefficient is 0.55 confirming non-universality and strong locality of such empirical equations. Interestingly, FZI* had a similar $R^{2}$ while FZI resulted in the lowest $R^{2}=0.20$. 


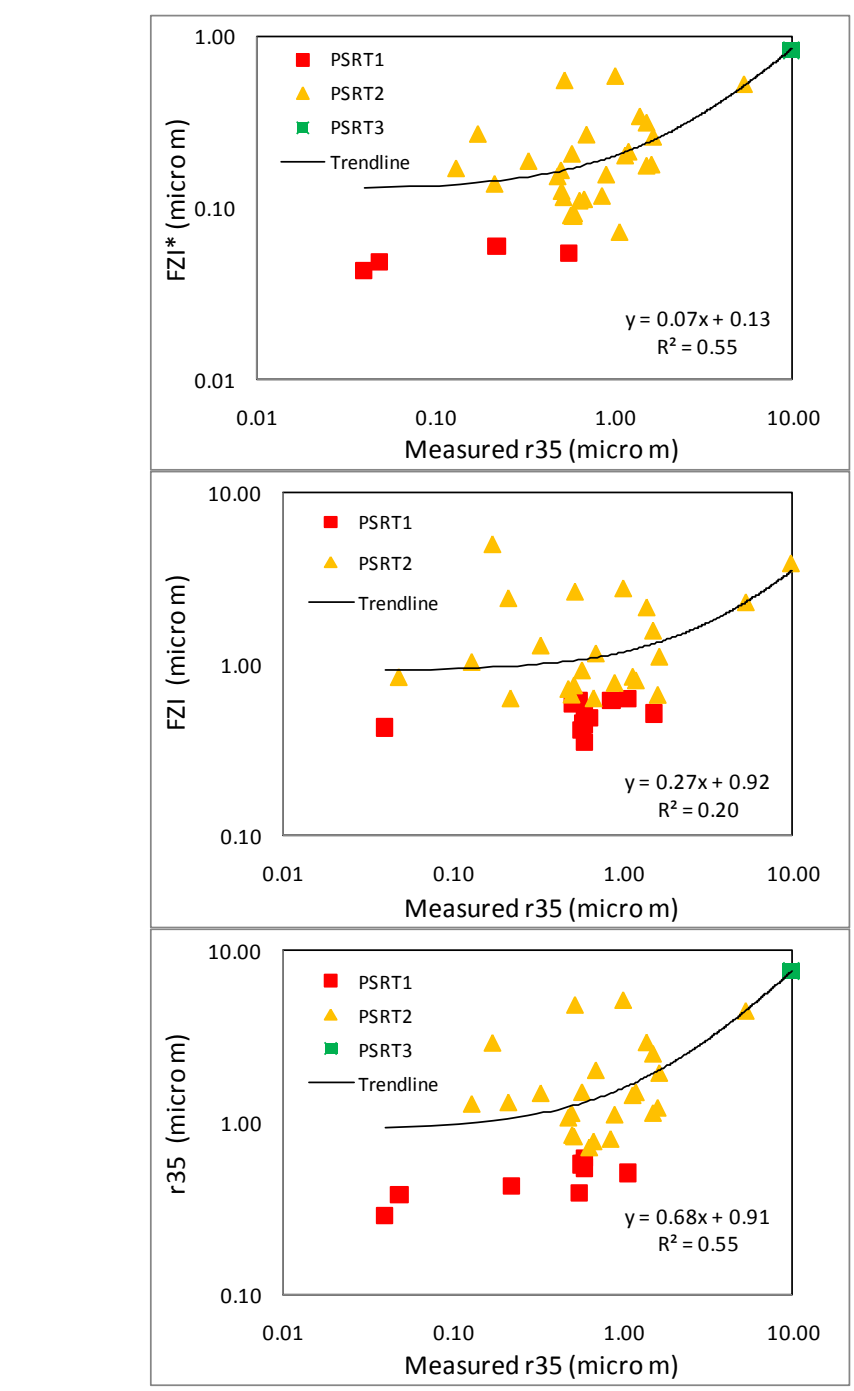

Figure 10 The relationship between different indices and the measured r35

\subsubsection{Water-oil primary drainage capillary pressure data}

We evaluate the accuracy of FZI*, FZI, Winland r35 and also MFZI to identify PSRTs. Sample properties are summarized in Supplementary Material. FZI*, FZI, Winland r35 and MFZI and the corresponding DRT numbers with $C_{4}=1.7$ for MFZI are calculated using the equations described previously. Figure 11 depicts the capillary pressure curves and the resulting PSRTs obtained by each method. FZI*, Winland r35 and MFZI provided us with three PSRTs while FZI identifies only two. Water-oil primary drainage capillary pressure experiments are performed by oil displacing water from the properly cleaned $100 \%$ water-saturated samples until the irreducible water saturation is achieved. Generally, rocks with lower PSRT values (i.e., tighter rocks with low permeability) should exhibit higher irreducible water saturations than those with higher PSRT values. Similarly, it is found from capillary pressure curves that tighter PSRTs 
yield higher water saturations. In another words, at a given water saturation, tighter PSRTs require higher capillary pressure values. Such phenomenon leads to water-oil primary drainage capillary pressure curves of tighter PSRTs to position on the right side of the higher DRT values of PSRTs. Figure 11 proves a better performance of FZI* in identification of PSRTs compared to other methods. It is expected that rocks within PSRT of DRT=1 (red color) appear at the right side of other two PSRTs, DRT=3 (green color) on the left side of the plot and DRT=2 (orange) in-between. It was observed that FZI and MFZI fail in identification of PSRTs and the predicted PSRTs do not form distinct clusters. Also, MFZI performed significantly poorer than FZI. The Winland r35 index resulted in a better data clustering than FZI and MFZI, but inferior than FZI*. Similar to the MICP case, although FZI* generates a considerably better result than other techniques, its ability to identify PSRTs was not as expected.
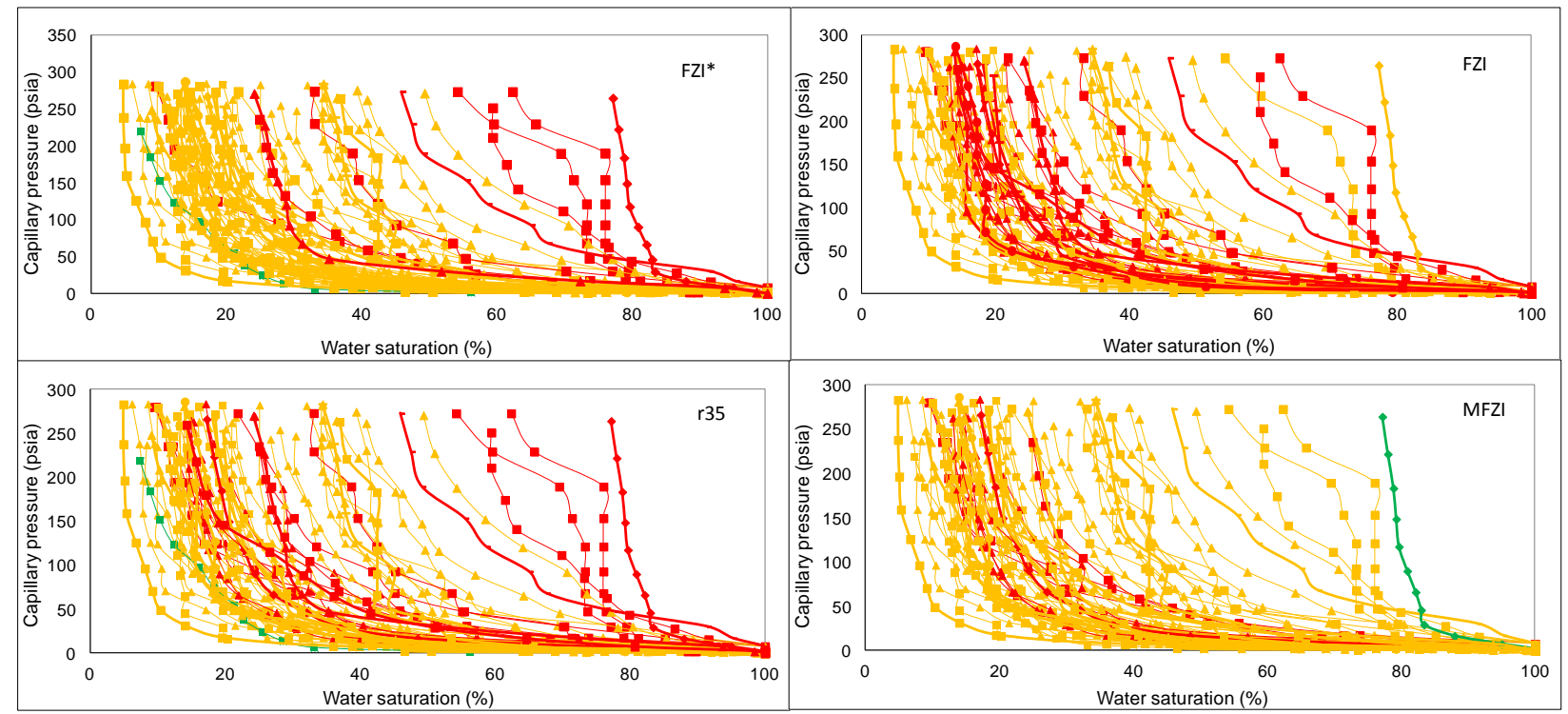

Figure 11 The water-oil primary drainage capillary pressure curves and the resulting PSRTs by FZI* (top left), FZI (top right), MFZI (bottom right) and Winland r35 (bottom left)

To study the relationship between different indices and irreducible water saturation, the plots in Figure 12 are generated. As stated before, for similar wettability tighter rocks have a greater irreducible water saturation. Thus, it is predictable that as irreducible water saturation increases, the indices decrease. This was only observed for FZI* and Winland r35 with correlation coefficients of 0.24 and 0.17 , respectively. MFZI exhibited a reverse (increasing) trend than FZI* and winland r35. 


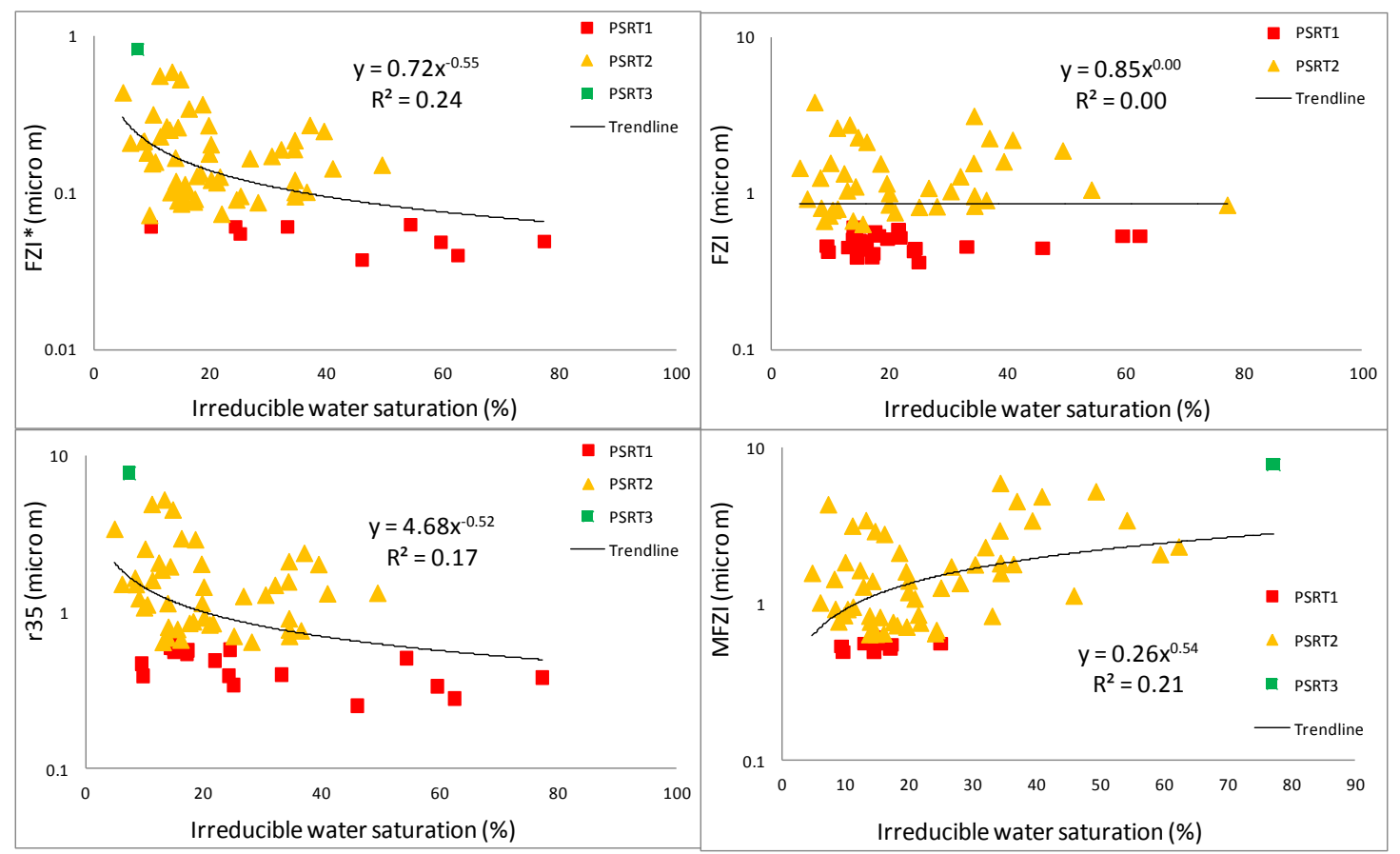

Figure 12 The relationship between irreducible water saturation and FZI* (top left), FZI (top right), MFZI (bottom right) and Winland r35 (bottom left)

To further investigate the relationship between different indices and capillary pressure curves, a statistical approach was employed. Our goal was to understand the main parameters affecting capillary pressure data. Logarithmic, power law and exponential models were fitted to the capillary pressure data and the highest $R^{2}$ values were observed for the case of the exponential model.

$$
\mathrm{P}_{\mathrm{c}}=\mathrm{C}_{5} \mathrm{e}^{-\mathrm{C}_{6}\left(\frac{\mathrm{S}_{\mathrm{w}}-\mathrm{S}_{\mathrm{wc}}}{1-\mathrm{S}_{\mathrm{wc}}}\right)}
$$

\section{Equation 23}

In the above equation $\mathrm{C}_{5}$ and $\mathrm{C}_{6}$ are the constants controlling the capillary pressure curve, and $\mathrm{S}_{\mathrm{w}}$ and $\mathrm{S}_{\mathrm{wc}}$ both in fraction are water saturation and irreducible water saturation, respectively. See Supplementary Material for the values of these constants and $R^{2}$ values for each sample. Based on the above equation it is expected that tight formations to have larger $\mathrm{C}_{5}$ and smaller $\mathrm{C}_{6}$ values. Figure 13 is the plot of different indices vs. $\mathrm{C}_{5}$. It is found that FZI*, FZI and Winland r35 decrease as $C_{5}$ increases. On the contrary, MFZI increases when $C_{5}$ increases. The coefficient of correlation was found $0.29,0.23,0.05$, and 0.02 for FZI*, Winland r35, MFZI and FZI, respectively. Figure 14 shows the plots of different indices vs. $\mathrm{C}_{6}$ where all increase as $\mathrm{C}_{6}$ 
increases. The coefficient of correlation was found $0.29,0.28,0.24$ and 0.16 for Winland r35, FZI*, FZI and MFZI, respectively.

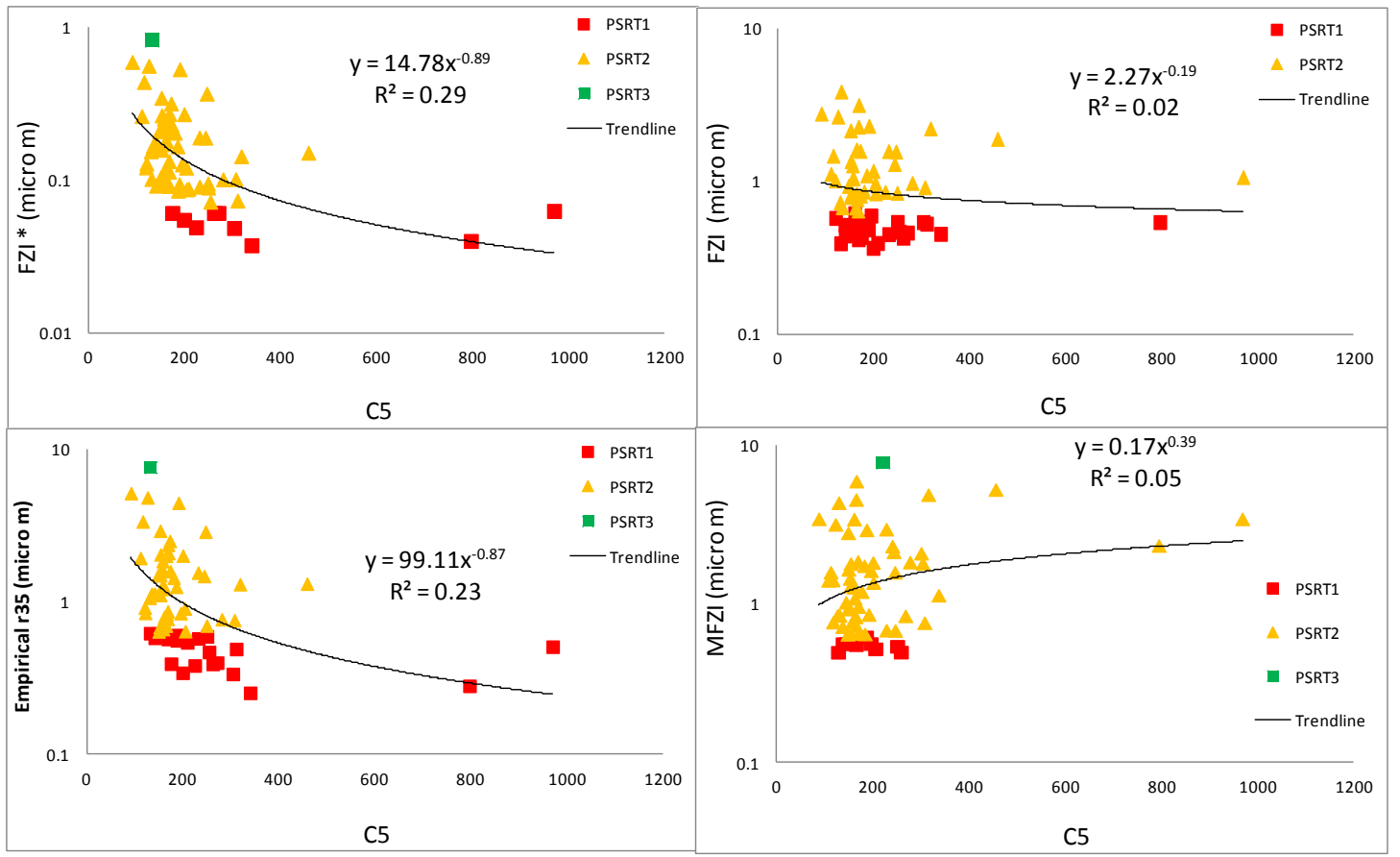

Figure 13 Different indices vs. C5.FZI* (top left), FZI (top right), MFZI (bottom right) and Winland r35 (bottom left)
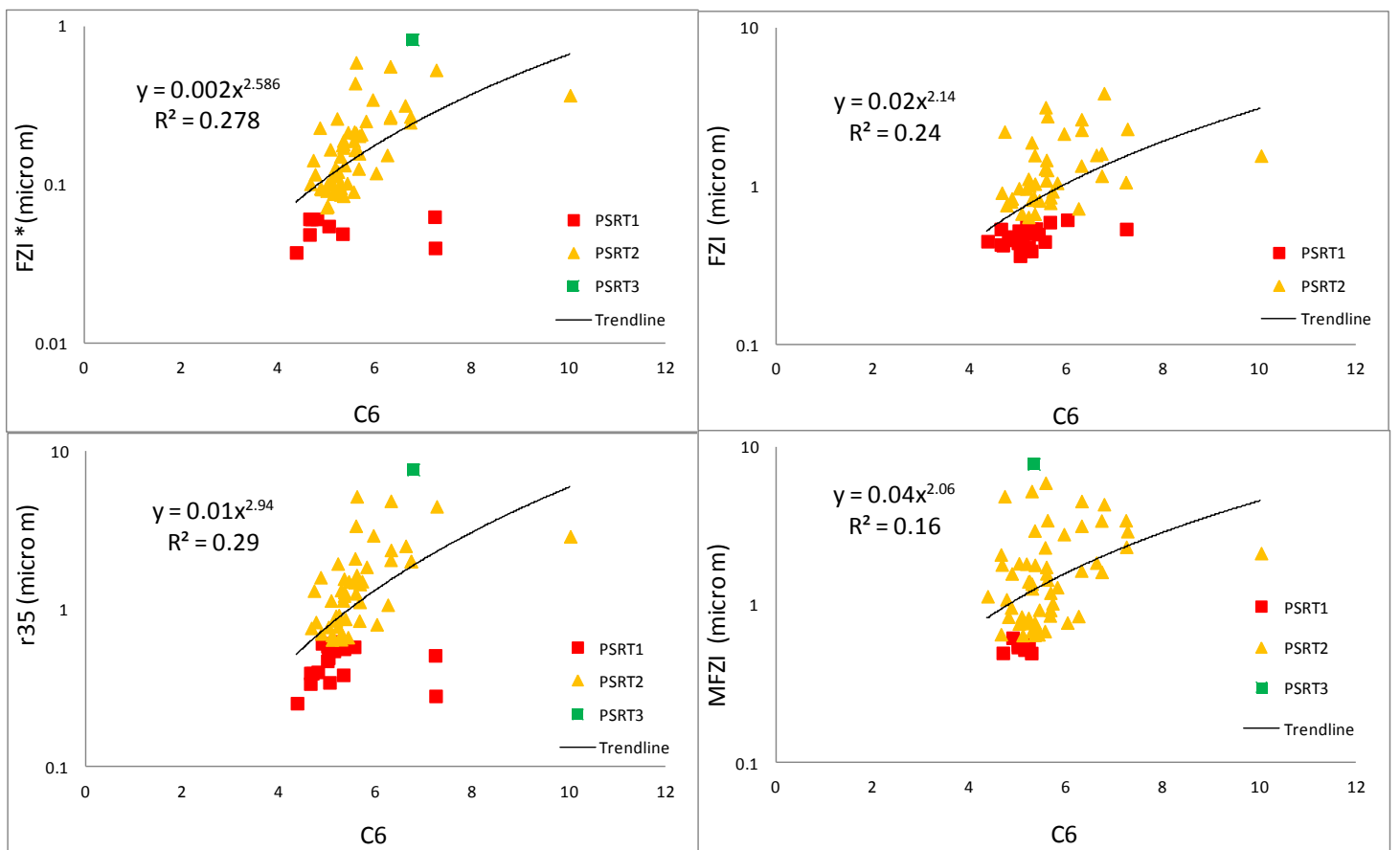

Figure 14 Different indices vs. C6. FZI* (top left), FZI (top right), MFZI (bottom right) and Winland r35 (bottom left)

\subsubsection{PDRTs}


To compare different indices in characterizing PDRTs, water-oil relative permeability experiments were carried out. Sample properties, FZI*, FZI, Winland r35 and MFZI and DRT values are calculated and presented in Supplementary Material. As explained earlier, PDRT is defined as a collection of rocks having similar fluid flow characteristics. Thus when dealing with water-oil displacement experiments, keeping all other factors constant, rocks within one PDRT must have similar $\frac{k_{e w}}{\phi}$ (or $\frac{k k_{r w}}{\phi}$ ) and $\frac{k_{e o}}{\phi}$ (or $\frac{k k_{r o}}{\phi}$ ) values. Moreover, rocks with a higher DRT number should appear on the upper region of $\frac{k_{e w}}{\phi}$ and $\frac{k_{e o}}{\phi}$ vs. water saturation cross-plots. Figure 15 and Figure 16 display PDRTs by each method using effective permeability data. FZI* and Winland r35 predicted three PDRTs whereas FZI and MFZI yielded two. FZI* showed the best performance in data clustering especially with water effective permeability. This can be due to the oil-wet characteristic of larger pores. In Winland r35, PDRT 1 and PDRT2 overlapped partially, especially with oil effective permeability data. FZI and MFZI did not generate any distinct PDRT.
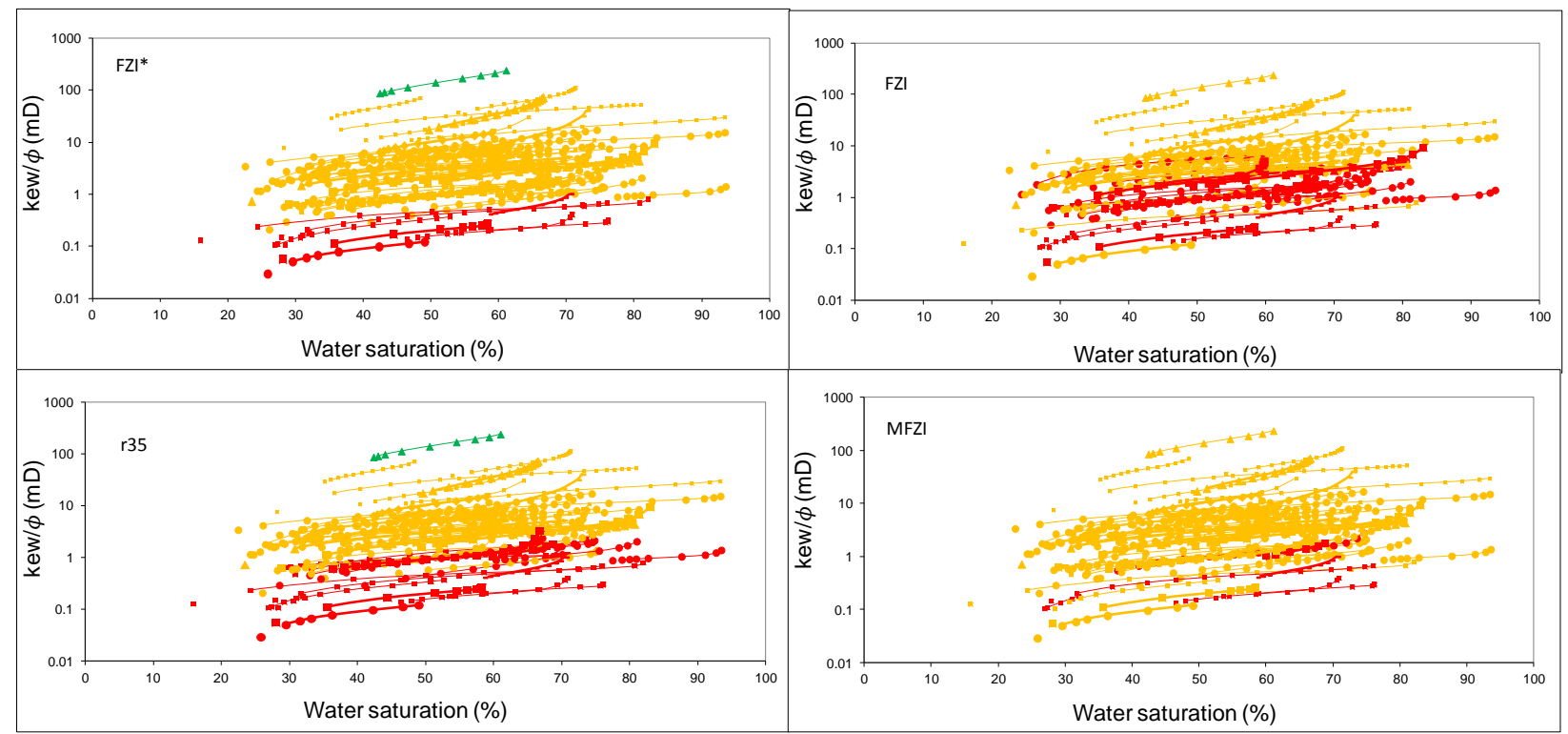

Figure 15 The $\frac{k_{\mathrm{ew}}}{\phi}$ vs. water saturation curves and the resulting PDRTs by FZI* (top left), FZI (top right), MFZI (bottom right) and Winland $\mathrm{r} 35$ (bottom left) 

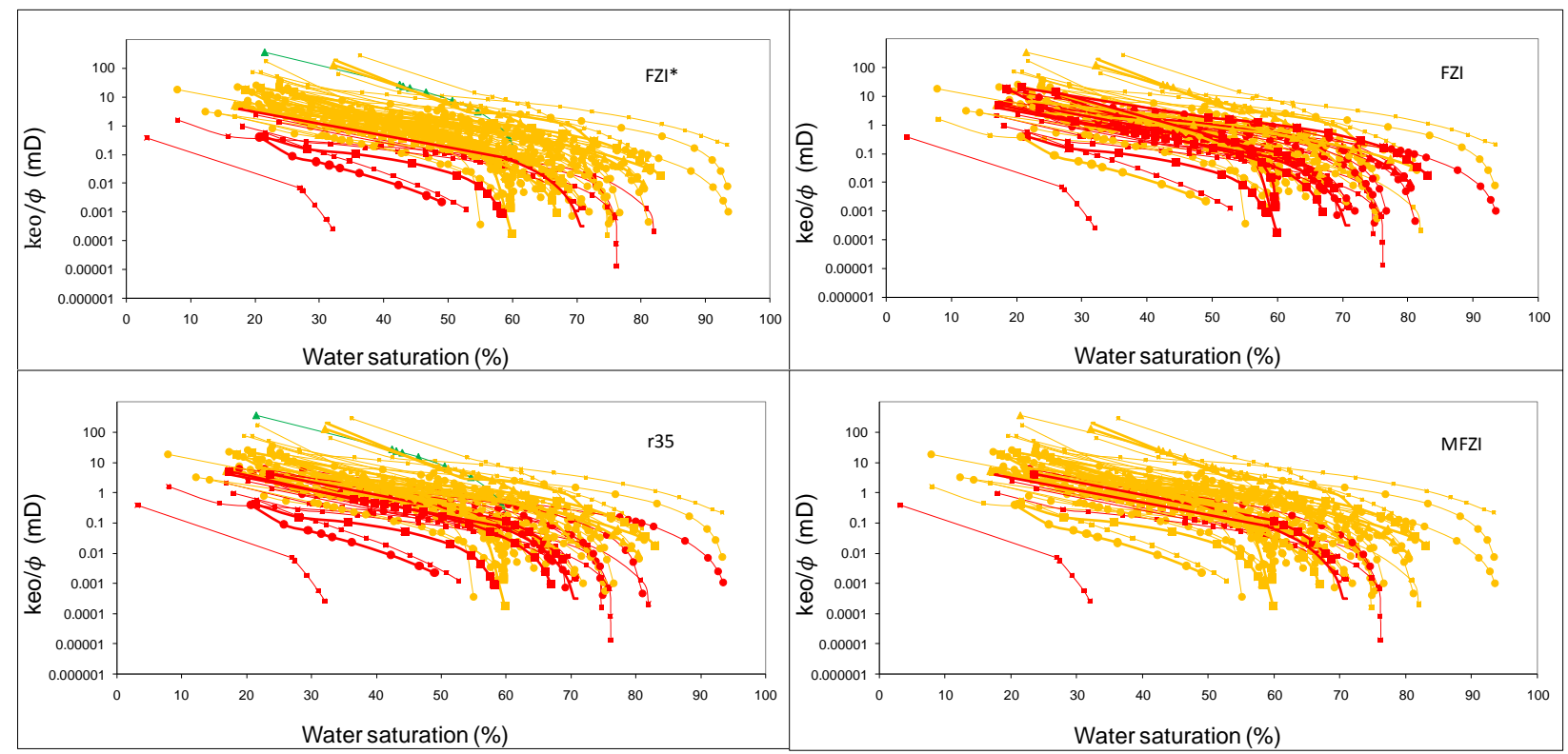

Figure 16 The $\frac{k_{\mathrm{eo}}}{\phi}$ vs. water saturation curves and the resulting PDRTs by FZI* (top left), FZI (top right), MFZI (bottom right) and Winland $\mathrm{r} 35$ (bottom left)

Effective permeabilities at $S_{w c}$, residual oil saturation $\left(S_{o r}\right)$, and $\left(\frac{k_{e}}{\phi}\right)_{w}=\left(\frac{k_{e}}{\phi}\right)_{o}$ points reflect pore geometry. Hence, their relationship with different indices can be investigated further. These data points are also presented in Supplementary Material for each sample. Figure 17 depicts different indices vs. $\frac{k_{e w}}{\phi}$ at $S_{\text {or }}$. In terms of the correlation coefficients, FZI* has the highest $R^{2}$ of 0.91 , followed by $0.89,0.57$ and 0.50 for Winland r35, MFZI and FZI, respectively. Furthermore, we realized FZI* outperforms other methods by generating distinct PDRTs. 


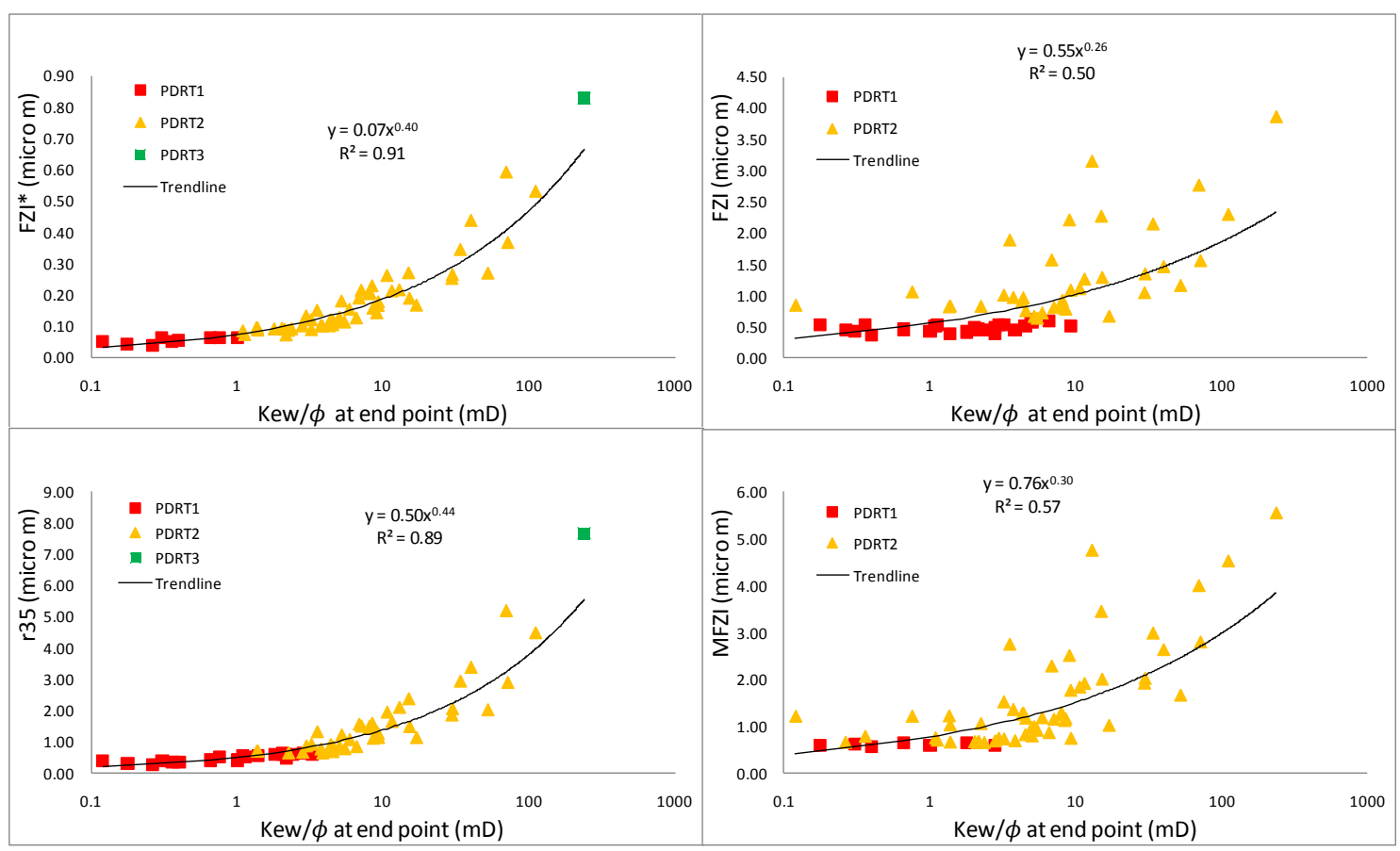

Figure 17 The relationship between different indices and the $\frac{\mathrm{k}_{\mathrm{ew}}}{\phi}$ at $S_{\mathrm{or}}$. FZI* (top left), FZI (top right), MFZI (bottom right) and Winland r35 (bottom left)

The plot of different indices vs. $\frac{k_{e o}}{\phi}$ at $S_{w c}$ is displayed in Figure 18. The correlation coefficients were calculated $0.90,0.85,0.42$ and 0.50 for FZI*, Winland r35, FZI and MFZI, respectively, which confirms the best performance by FZI* compared to other methods. In addition, all indices were plotted vs. $\frac{k_{e}}{\phi}$ at the cross-over saturation point in Figure 19. FZI* with the highest $R^{2}$ of 0.86 provided us with the best PDRTs in comparison to Winland r35, FZI and MFZI with correlation coefficients of $0.84,0.49$ and 0.55 , respectively. 


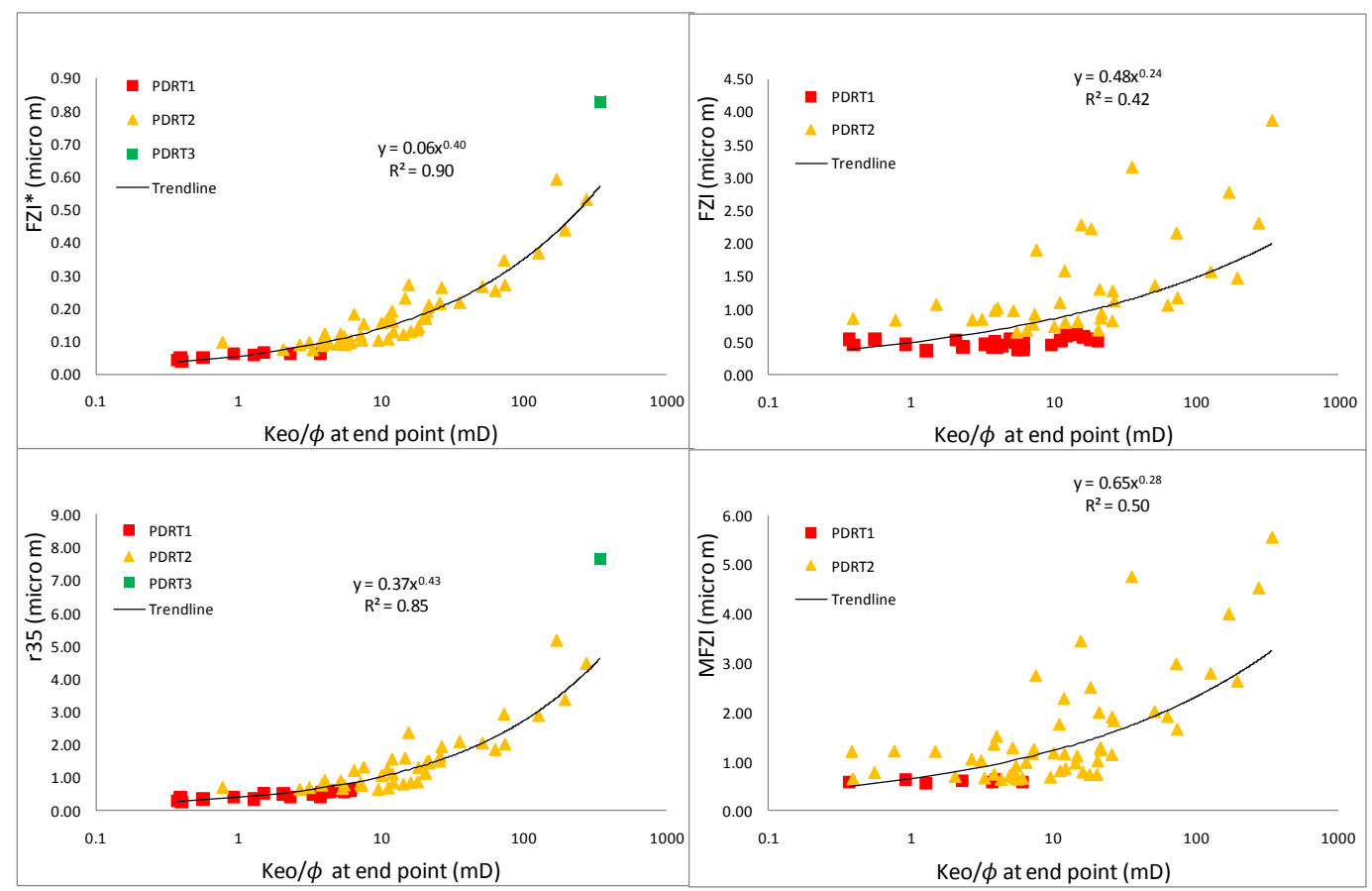

Figure 18 The relationship between different indices and the $\frac{\mathrm{k}_{\mathrm{eo}}}{\phi}$ at $\mathrm{S}_{\mathrm{wc}}$. FZI* (top left), FZI (top right), MFZI (bottom right) and Winland r35 (bottom left)

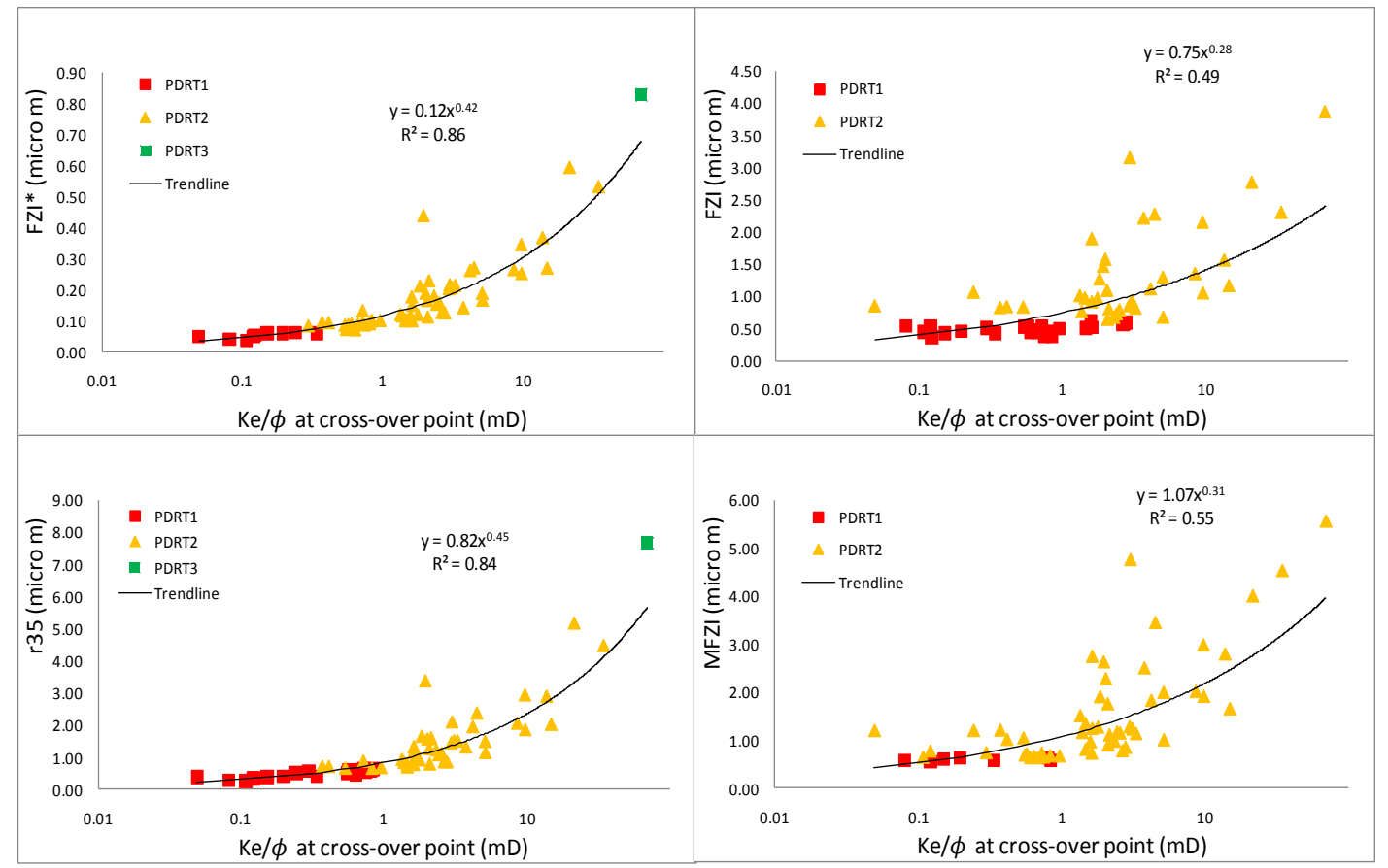

Figure 19 The relationship between different indices and the $\frac{k_{\mathrm{e}}}{\phi}$ at cross-over point. FZI* (top left), FZI (top right), MFZI (bottom right) and Winland $\mathrm{r} 35$ (bottom left) 
We studied the relationship between different indices and parameters governing on water-oil $\frac{k_{e}}{\phi}$ curves. We found that power-law and logarithmic functions will result in the best $R^{2}$ values for $\frac{k_{e w}}{\phi}$ and $\frac{k_{e o}}{\phi}$, correspondingly. The equations of these functions are as follows:

$$
\frac{\mathrm{k}_{\mathrm{ew}}}{\phi}=\mathrm{C}_{7}\left(\frac{\mathrm{S}_{\mathrm{w}}-\mathrm{S}_{\mathrm{wc}}}{1-\mathrm{S}_{\mathrm{or}}-\mathrm{S}_{\mathrm{wc}}}\right)^{\mathrm{C} 8}
$$

Equation 24

$$
\frac{\mathrm{k}_{\mathrm{eo}}}{\phi}=\mathrm{C}_{9} \operatorname{Ln}\left(\frac{\mathrm{S}_{\mathrm{w}}-\mathrm{S}_{\mathrm{wc}}}{1-\mathrm{S}_{\mathrm{or}}-\mathrm{S}_{\mathrm{wc}}}\right)
$$

\section{Equation 25}

in which $\mathrm{C}_{7}, \mathrm{C}_{8}$ and $\mathrm{C}_{9}$ are constants. The values of $\mathrm{C}_{7}$ and $\mathrm{C}_{9}$ and the associated $R^{2}$ values can be found in Supplementary Material. We were not able to delineate any meaningful trend between $C_{8}$ and the indices. Figure 20 is the indices vs. $C_{7}$ and shows they increase as $C_{7}$ increases. The coefficient of correlation was calculated $0.91,0.89,0.59$, and 0.52 for FZI*, Winland r35, MFZI and FZI, respectively. Figure 21 is the cross-plot of different indices vs. $\mathrm{C}_{9}$. The indices increase as $C_{9}$ increases with correlation coefficients of $0.84,0.80,0.45$ and 0.38 for FZI*, Winland r35, MFZI, and FZI, correspondingly.

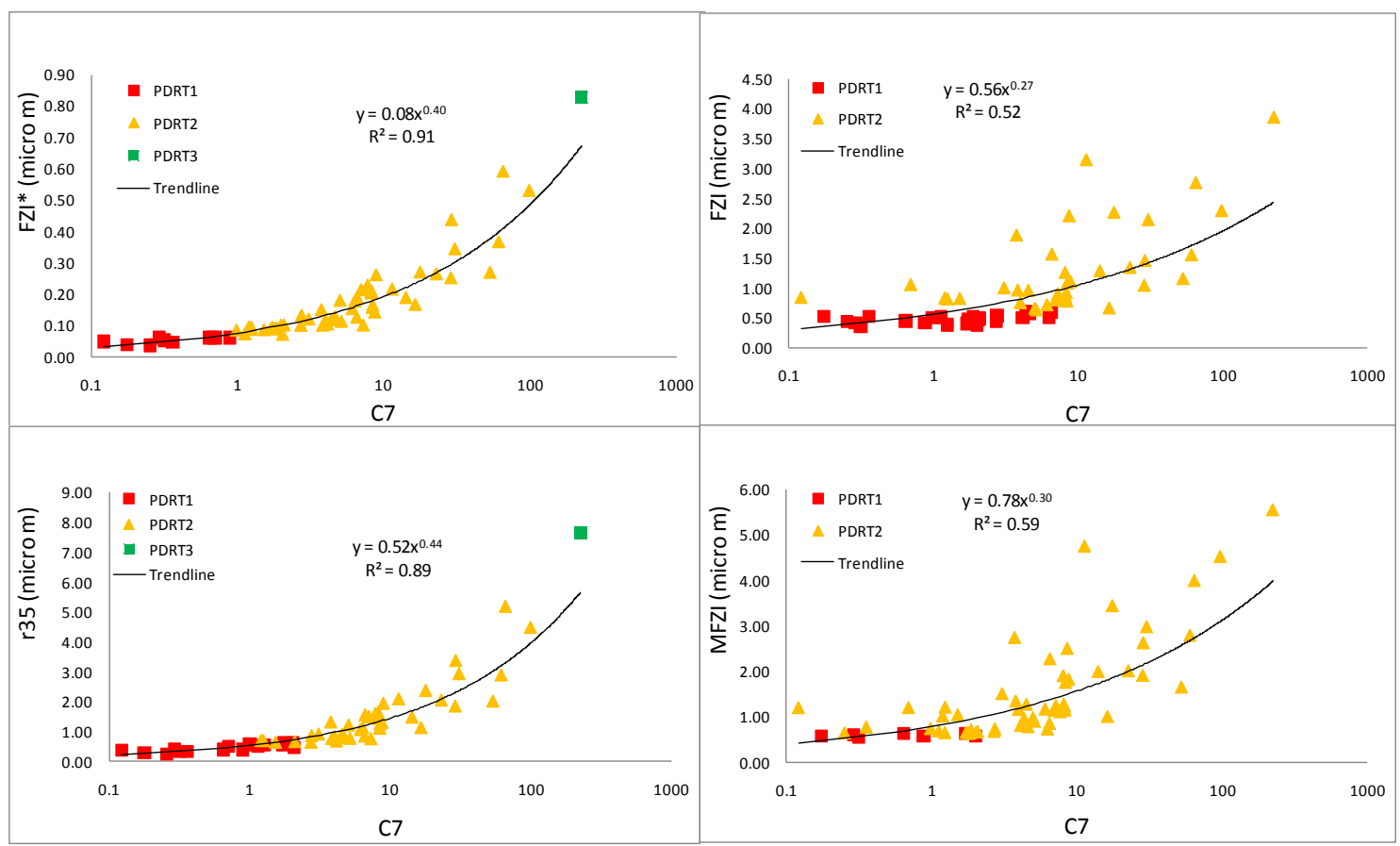

Figure 20 Different indices vs. C7. FZI* (top left), FZI (top right), MFZI (bottom right) and Winland r35 (bottom left) 


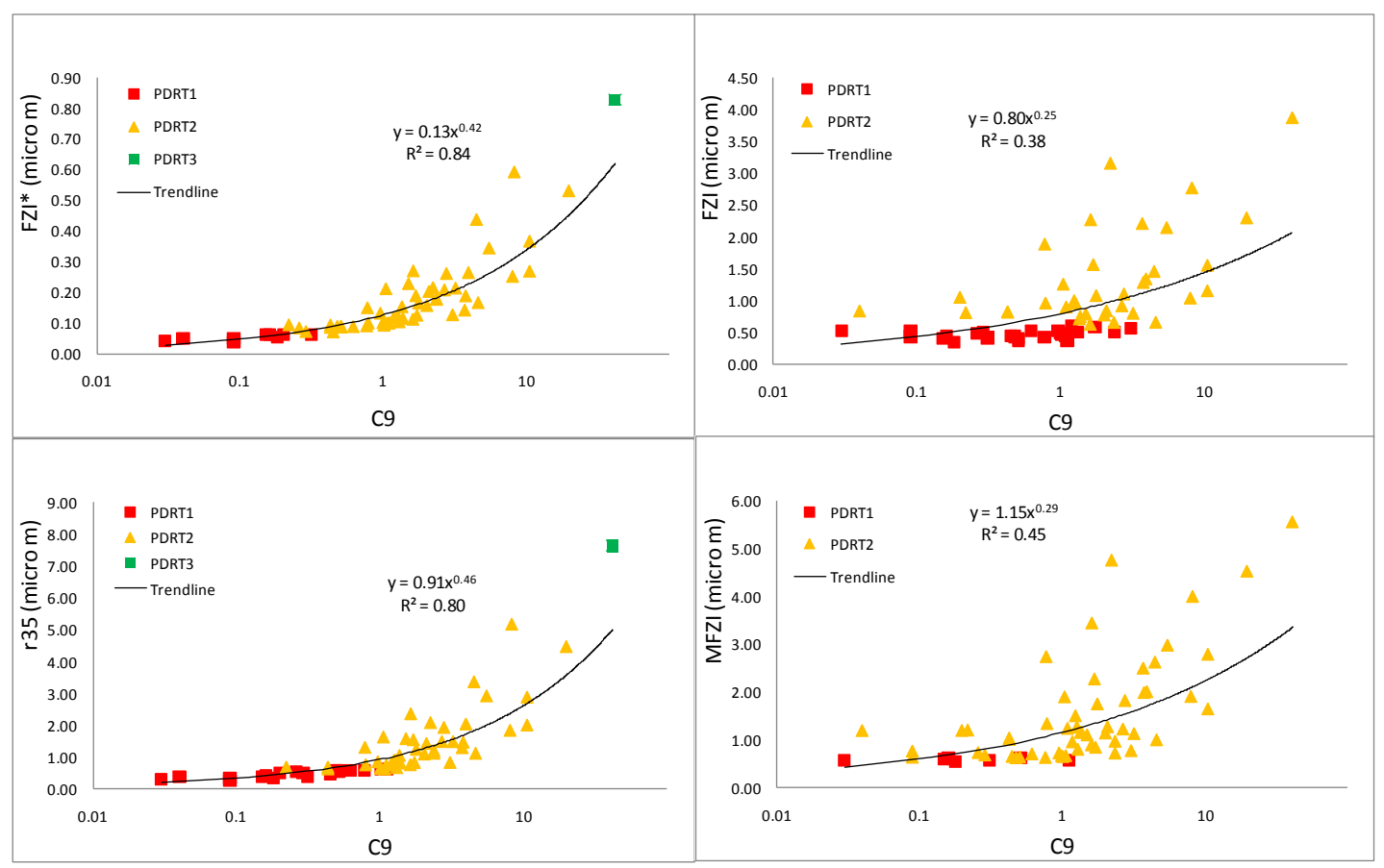

Figure 21 Different indices vs. C9. FZI* (top left), FZI (top right), MFZI (bottom right) and Winland r35 (bottom left)

\section{Discussion}

\section{Geological significance of FZI*}

In clastic rocks, when the initial state of pores is not altered by diagenesis, grains will be subspherical. In this regard, when the grains are larger, both pores and pore throat sizes increase (Rashid et al., 2015). However, carbonates will undergo a significant level of diagenesis which modifies original intact pore networks. This will make proposing a unique relationship between a grain size and a pore size/pore throat size very difficult. Based on our theoretical analysis, FZI that is derived from the general form of the Kozeny-Carman equation contains the term that represents a grain diameter, $D_{p}$, whereas fluid flow is not directly related to solid matrix properties. On the contrary, FZI* contains the properties of a pore network which makes it superior to FZI. The Nooruddin and Hussain (2011) and Izadi and Ghalambor (2013) models are also based on the general form of the Kozeny-Carman equation, which makes them venerable to this problem. Additionally, the model by Nooruddin and Hussain (2011) does not consider the tortuosity properly in determining the rock types. It should also be noted that the Nooruddin and Hussain (2011) model is more complicated than the original FZI approach considering its dependency on a cementation factor, which usually needs SCAL measurements. In addition, the 
connate water saturation which is a function of wettability may have an adverse effect on evaluating a petrophysical rock type developed by Izadi and Ghalambor (2013).

\section{Effective porosity vs. connected porosity}

In a porous medium where a variety of pore throat sizes exist, only a portion of the total connected pore spaces effectively contributes to the flow. This portion constitutes a class of larger pore throats and causes other smaller pore throats to have a minimal impact on permeability. If the porosity that is derived from the total connected pore spaces is called connected porosity, then the portion of this connected porosity that has a role in fluid flow is effective porosity. This is the porosity that is considered in flow equations and FZI*. Though, connected porosity instead of effective porosity is usually measured in the lab. This problem can be addressed if MICP data are acquired (Rabiller, 2017). In carbonate rocks such substitution of porosity values may introduce a significant error to the results due to the strong influence of diagenesis on pore-network geometry and heterogeneity. This can be one of the reasons of abnormal MICP and water-oil primary drainage capillary pressure data when clustering with FZI*. Besides, another main factor that may cause such deviation is that FZI $^{*}$ was employed instead of PSRTI to delineate PSRTs. This means the term $\sqrt{F_{s} \tau}$ was not considered in evaluations.

\section{$\underline{\text { Relative permeability curves }}$}

A common understanding in rock typing studies has historically been that rocks within one PDRT should exhibit similar relative permeability $k_{r}$ data (i.e., $k_{r}$ vs $S_{w}$ curves). Through an extensive search in the literature, one cannot find any scientific reasoning for it. Additionally, since relative permeability is a normalized parameter and varies between zero and one, establishing a robust relationship between PDRTs and relative permeability will become very challenging (see Figure 22). Our analysis shows that $\frac{k_{e}}{\phi}$ vs $S_{w}$ should be plotted to reveal different rock type clusters. For the samples studied here, a mixed-wet characteristic was observed. In this case, smaller pores remain water-wet while larger oil-wet pores will control the flow. Finally, we assumed all rocks studied are oil-wet, which is not an incorrect assumption, considering the oilwet nature of most carbonate rocks (Mirzaei-Paiaman et al., 2013). 


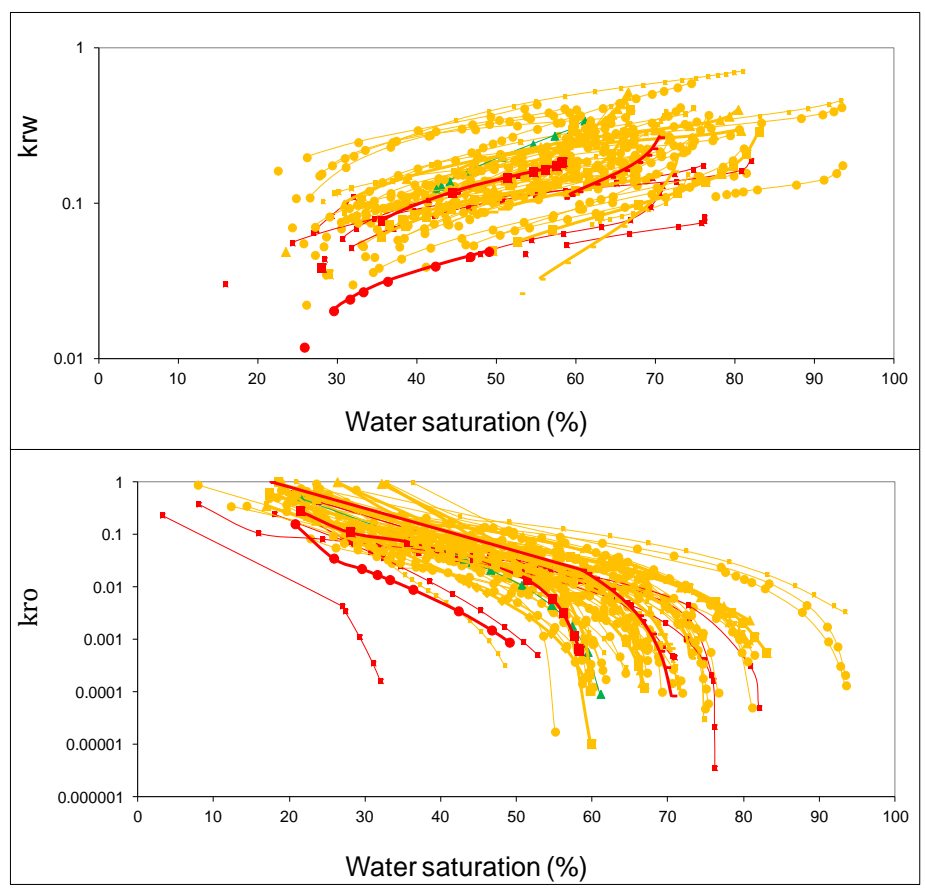

Figure 22 The krw and kro vs. water saturation curves and the resulting PDRTs by FZI*

\section{$\underline{\text { J-function and its modification }}$}

One of the main contributions of this study is proposing a modification for the conventional Jfunction. So far, it's believed that $\frac{k}{\phi}$ in the J-function is sufficient to model rock microstructure to normalize the capillary pressure data. However, researchers have pointed out that the Jfunction is not recommended for carbonate rock characterization and have proposed additional refinements and restrictions (e.g., applying lithology type and texture information) (Brown, 1951, Rebelle et al., 2014). This denotes that an additional term should be added to the J-function to enhance its applicability in characterizing microstructures and normalizing capillary pressure data. Our analysis also showed that when fluid and rock-fluid interaction properties remain constant, rocks with similar $\sqrt{\frac{\mathrm{k}}{\phi} \mathrm{F}_{\mathrm{s}} \tau}$ or $\mathrm{FZI}^{*} \sqrt{\mathrm{F}_{\mathrm{s}} \tau}$ will present similar $\mathrm{P}_{\mathrm{c}}$ behavior and constitute one PSRT. This is a strong indication that the J-function should be modified to include the term $\sqrt{F_{s} \tau}$. Saboorian-Jooybari et al. (2010) and then Ferreira et al. (2015) presented a modified Jfunction which incorporates a hydraulic tortuosity term, $\tau$. Considering what is argued, the modified J-function that is superior to the conventional one and suitable for variety of microstructures can be written as: 


$$
J=\frac{P_{c}}{\gamma \cos (\theta)} \sqrt{\frac{k}{\phi} F_{s} \tau}
$$

Equation 26

\section{Conclusions}

In this study, we studied static and dynamic rock types separately and showed that these two do not necessarily overlap and thus a new index should independently be developed to define each one. We proposed new rock typing indices that can be used for various petrophysical, drilling, production, injection, reservoir studies and simulation purposes. This also enabled us to modify the conventional J-function to enhance its capability in defining capillary pressure data from various rock types. The new models were validated with an extensive laboratory analysis including MICP, capillary pressure, and relative permeability data on core plugs from a carbonate reservoir. Based on data analysis and mathematical manipulations the following conclusions can be made:

- A PDRT can be defined as rock units with a similar fluid flow behavior. From the physical stand point, it can be referred to rocks under similar $\frac{\mathrm{v}}{\mathrm{dp} / \mathrm{dx}}$ conditions.

- An index for characterization of PDRTs, known as FZI*, can be developed by using either the fundamental form of the Kozeny-Carman equation or Darcy's law for single and multi-phase flow systems.

- A PSRT can be defined as those rocks types that have identical primary drainage capillary pressure data. This idea can be extended to presenting unique water saturation for a given height above the FWL.

- An index to study PSRTs, known as PSRTI, was developed by combining the YoungLaplace capillary pressure equation with the Kozeny-Carman expression.

- It can be concluded that by keeping all other petrophysical factors constant and only considering pore-network structure, PSRTs do not necessarily overlap with PDRTs. Depending on the complexity of pore-network geometry there may be a large discrepancy between these two definitions.

- From this study and based on an extensive comparison of existing models, it can be deduced that almost all empirical indices for rock typing have a similar mathematical 
format. They are defined in such a way to be considered as the special solution of a general universal equation of our proposed model.

- Using the $\mathrm{FZI}^{*}$ and without any prior knowledge of petrographic, depositional or diagenetic information the PDRTs and PSRTs, we were able to successfully characterize one of the most heterogeneous carbonate reservoirs in the southwest of Iran.

- The J-function was modified based on the new indices by adding microstructural parameters to better represent capillary pressure in highly heterogeneous rock types.

\section{Acknowledgement}

Abouzar Mirzaei-Paiaman thanks National Iranian South Oil Company (NISOC) and National Iranian Oil Company (NIOC) for permission to publish this work.

\section{References}

- Abbaszadeh, M., Fujii, H., Fujimoto, F., 1996. Permeability Prediction by Hydraulic Flow Units -Theory and Applications. SPE Formation Evaluation 11, 263-271.

- Aguilera, R. 2002. Incorporating capillary pressure, pore throat aperture radii, height above free-water table, and Winland r35 values on Pickett plots. AAPG Bulletin, v. 86, no. 4 (April 2002), pp. 605-624

- Aguilera, R., 2004, Integration of geology, petrophysics, and reservoir engineering for characterization of carbonate reservoirs through Pickett plots, AAPG Bulletin, v. 88, no. 4, p. 433-446.

- Amaefule, J.O., Altunbay, M., Tiab, D., Kersey, D.G., Keelan, D.K., 1993. Enhanced reservoir description using core and log data to identify hydraulic flow units and predict permeability in uncored intervals/wells. In: SPE Annual Technical Conference and Exhibition, 3-6 October, Houston, Texas.

- Archie, G. E., 1952, Classification of carbonate reservoir rocks and petrophysical considerations, AAPG Bulletin, v. 36, p. 278-298.

- Askari, A.A., Behrouz, T., 2011. A Fully Integrated Method for Dynamic Rock Type Characterization Development in One of Iranian Off-Shore Oil Reservoir. Journal of Chemical and Petroleum Engineering, University of Tehran, Vol. 45, No.2, PP. 83-96

- Brown, H.W. 1951. Capillary pressure investigations. Petroleum Transactions, AIME, vol. 192, 67-74.

- Cai, J., Perfect, E., Cheng, CL., Hu. X. 2014. Generalized modeling of spontaneous imbibition based on HagenPoiseuille flow in tortuous capillaries with variably shaped apertures. Langmuir, 30 (18), 5142-5151

- Carman, P.C., 1937. Fluid flow through granular beds. Trans. Inst. Chem. Eng. 15, 150-166.

- Chen, X., and Yao, G. 2017. An improved model for permeability estimation in low permeable porous media based on fractal geometry and modified Hagen-Poiseuille flow. Fuel 210, 748-757.

- Chen, X., and Zhou, Y. 2017. Applications of digital core analysis and hydraulic flow units in petrophysical characterization. Advances in Geo-energy Research. Vol. 1, No. 1, p. 18-30, doi: 10.26804/ager.2017.01.02.

- Chopra, A.K. 1988. Reservoir Descriptions Via Pulse Testing: A Technology Evaluation. SPE-17568-MS, International Meeting on Petroleum Engineering, 1-4 November, Tianjin, China. DOI

- Chopra, A.K., Stein, M.H., Ader, J.C. 1989. Development of reservoir descriptions to aid in design of EOR projects. SPE-16370-PA, SPE Reservoir Engineering 4(2), 143-150.

- Corbett, P.W.M., and Potter, D.K. 2004. Petrotyping: a basemap and atlas for navigating through permeability and porosity data for reservoir comparison and permeability prediction. International Symposium of the Society of Core Analysts held in Abu Dhabi, UAE, 5-9 October, 2004. SCA2004-30

- Davies, D.K., Vessell, R.K. 1996. Identification and Distribution of Hydraulic Flow Units in a Heterogeneous Carbonate Reservoir: North Robertson Unit, West Texas. SPE-35183-MS. DOI https://doi.org/10.2118/35183MS

- Donaldson, E.C., Ewall, N., and Singh, B. 1991. Characteristics of capillary pressure curves. Journal of Petroleum Science and Engineering/ Volume 6, Issue 3, November 1991, Pages 249-261. 
- Dunham, R. J., 1962, Classification of carbonate rocks according to depositional texture, in W. E. Ham, ed., Classification of carbonate rocks, American Association of Petroleum Geologists Memoir 1, p. 108-121

- Ferreira, F.C., Booth, R., Oliveira, R., Carneiro, G., Bize-Forest, N., and Wahanik, H. 2005. New RockTyping Index Based on Hydraulic and Electric Tortuosity Data for Multi-Scale Dynamic Characterization of Complex Carbonate Reservoirs. SPE-175014-MS, SPE Annual Technical Conference and Exhibition, 28-30 September, Houston, Texas, USA. DOI https://doi.org/10.2118/175014-MS

- Gholami, V.,Mohaghegh, S., 2009. Intelligent Upscaling of Static and Dynamic Reservoir Properties. SPE124477-MS, SPE Annual Technical Conference and Exhibition, 4-7 October, New Orleans, Louisiana.

- Izadi, M., Ghalambor, A., 2013. New approach in permeability and hydraulic-flow unit determination. SPE Reserv. Eval. Eng. 16 (3), 257-264.

- Jaya, I., Sudaryanto, A., Widarsono, B. 2005. Permeability Prediction Using Pore Throat and Rock Fabric: A Model from Indonesian Reservoirs. SPE-93363-MS, SPE Asia Pacific Oil and Gas Conference and Exhibition, 5-7 April, Jakarta, Indonesia. DOI https://doi.org/10.2118/93363-MS

- Jongkittinarukorn, K., and Tiab, D. 1997. Identification of flow units in shaly sand reservoirs. Journal of Petroleum Science and Engineering 17, 237-246.

- Katz, A.J., and Thompson, A.H. 1986. Quantitative prediction of permeability and electrical conductivity in porous rock. SEG Technical Program Expanded Abstracts 1986: pp. 6-7. DOI

- Kolodzie, S. 1980. Analysis of pore throat size and use of the Waxman-Smits equation to determine OOIP in Spindle field, Colorado. SPE-9382-MS, SPE Annual Technical Conference and Exhibition, 21-24 September, Dallas, Texas. DOI https://doi.org/10.2118/9382-MS

- Kozeny, J., 1927. ber kapillare Leitung des Wassers im Boden, Sitzungsberichte.Royal Academy of Science Vienna, Proc. Class I, 136, 271-306.

- Kwon, B. S., and G. R. Pickett, 1975, A new pore structure model and pore structure interrelationships: Society of Professional Well Log Analysts 16th Annual Logging Symposium, 4-7 June 1975, pages 1-14.

- $\quad$ Lin, B., Chen, M., and Pang, H. 2015. Modeling pore size distribution of southern Sichuan shale gas reservoirs. Journal of Natural Gas Science and Engineering Volume 26, Pages 883-894.

- Mirzaei-Paiaman A, Saboorian-Jooybari H. 2016. A method based on spontaneous imbibition for characterization of pore structure: application in pre-SCAL sample selection and rock typing. J Nat Gas Sci Eng 35:814-25. http://dx.doi.org/10.1016/j.jngse.2016.09.023.

- Mirzaei-Paiaman, A., Saboorian-Jooybari, H., Pourafshari, P., 2015. Improved method to identify hydraulic flow units for reservoir characterization. Energy Technol. 3 (7), 726-733.

- Mirzaei-Paiaman, A., Masihi, M., Standnes, D.C. (2013) Index for characterizing wettability of reservoir rocks based on spontaneous imbibition recovery data. Energy Fuels 27: 7360 -7368, DOI: dx.doi.org/10.1021/ef401953b

- Ngo, V.T., Lu, V.D., Nguyen, M.H., Hoang, T.M., Nguyen, H.M., and Le, V.M. 2015. A Comparison of Permeability Prediction Methods Using Core Analysis Data. SPE-175650-MS, SPE Reservoir Characterisation and Simulation Conference and Exhibition, 14-16 September, Abu Dhabi, UAE.

- Nooruddin, H., Hossain, M., 2011. Modified Kozeny-Carmen correlation for enhanced hydraulic flow unit characterization. J. Petroleum Sci. Eng. 80, 107-115.

- Oliveira, G.P., Roque, W.L., Araújo, E.A., Diniz, A.A.R., Simões, T.A., Santos, M.D. 2016. Competitive placement of oil perforation zones in hydraulic flow units from centrality measures. Journal of Petroleum Science and Engineering, 147, 282-291. DOI https://doi.org/10.1016/j.petrol.2016.06.008

- Pittman, E. D., 1992, Relationship of porosity and permeability to various parameters derived from mercury injection-capillary pressure curves for sandstone: AAPG Bulletin, 76, 191-198.

- Purcell, W. R., 1949, Capillary pressures-their measurement using mercury and the calculation of permeability therefrom, American Institute of Mechanical Engineers, Petroleum Transaction, AIME, T.P. 2544, pages 39-48.

- Rabiller, P., 2017. Combining porosimetry and Purcell permeability modeling to calibrate FZI and define a dynamic permeability cut-off. International Symposium of the Society of Core Analysis, Vienna, Austria, 27-30 August 2017.

- $\quad$ Rashid, F., Glover, P.W.J., Lorinczi, P., Hussein, D., Collier, R., Lawrence, J. 2015. Permeability prediction in tight carbonate rocks using capillary pressure measurements. Marine and Petroleum Geology 68, 536-550.

- Rebelle, M. 2014. Rock-typing In Carbonates: A Critical Review Of Clustering Methods. SPE-171759-MS, Abu Dhabi International Petroleum Exhibition and Conference, 10-13 November, Abu Dhabi, UAE. 
- Roque, W.L., Oliveira, G.P., Santos, M.D., and Simões, T.A. 2017. Production zone placements based on maximum closeness centrality as strategy for oil recovery. Journal of Petroleum Science and Engineering, 156, 430-441. DOI: 10.1016/j.petrol.2017.06.016

- Saboorian-Jooybari, H., 2017. A structured mobility-based methodology for quantification of net-pay cutoff in petroleum reservoirs. SPE-183643-PA. SPE Reservoir Evaluation \& Engineering, 20 (02), 1-17.

- Saboorian-Jooybari, H., Mowazi, G.H., Jaberi, S.R., 2010. A New Approach for Rock Typing Used in One of the Iranian Carbonate Reservoir (A Case Study). In: Paper SPE 131915 Presented at the International Oil and Gas Conference and Exhibition, Beijing, China, 8-10 June.

- Serag El Din, S., Dernaika, M.R., and Kalam, Z. 2014. Integration of Petrophysical SCAL Measurements for Better Understanding Heterogeneity Effects in Carbonates: Case Study Using Samples from a Super Giant Field in Abu Dhabi. IPTC-17572-MS, International Petroleum Technology Conference, 19-22 January, Doha, Qatar.

- Shen, L. and Chen, Z. 2007. Critical review of the impact of tortuosity on diffusion, Chemical Engineering Science 62, 3748-3755.

- Siddiqui, S., Okasha, T.M., Funk, J.J., and Al-Harbi, A.M. 2006. Improvements in the selection criteria for the representative special core analysis samples. SPE-84302-PA, SPE Reservoir Evaluation \& Engineering, 9 (6), 647-653. DOI https://doi.org/10.2118/84302-PA

- Sokhal, A., Benmalek, S., and Ouadfeul, S. 2016. Rock Type and Permeability Prediction using Flow Zone Indicator with an application to Berkine Basin (Algerian Sahara)., SEG International Exposition and 86th Annual Meeting, 16-21 October, Dallas, Texas. Pages 3068-3072

- Soto, R.B., Garcia, J.C. Torres, F., and Perez, G.S. 2001. Permeability Prediction Using Hydraulic Flow Units And Hybrid Soft Computing Systems. SPE-71455-MS, SPE Annual Technical Conference and Exhibition, 30 September-3 October, New Orleans, Louisiana. DOI https://doi.org/10.2118/71455-MS

- Tabatabaei, S.M.E., Kadkhodaie-Ilkhchi, A., bHosseini, Z., bAsghari Moghaddam, A., 2015. A hybrid stochastic-gradient optimization to estimating total organic carbon from petrophysical data: A case study from the Ahwaz oilfield, SW Iran. Journal of Petroleum Science and Engineering, Volume 127, Pages 35-43.

- Taslimi, M., Bohloli, B., Kazemzadeh, E., and Kamali, M.R. 2008. Determining Rock Mass Permeability in a Carbonate Reservoir, Southern Iran Using Hydraulic Flow Units and Intelligent Systems. 2nd IASME / WSEAS International Conference on GEOLOGY and SEISMOLOGY (GES '08), Cambridge, UK, February 23-25. Pages 132-139

- Thomeer, J. H., 1960, Introduction of a pore geometrical factor defined by the capillary pressure curve, JPT, March, p. 73-77.

- Thomeer, J.H.M. 1960. Introduction of a Pore Geometrical Factor Defined by the Capillary Pressure Curve. J Pet Technol 12 (3): 73-77. SPE-1324-G. http://dx.doi.org/10.2118/1324-G

- Wang, F., Lian, P., Jiao, L., Liu, Z., Zhao, J., and Gao, J. 2018. Fractal analysis of microscale and nanoscale pore structures in carbonates using high-pressure mercury intrusion. Geofluids, available at https://www.hindawi.com/journals/geofluids/aip/4023150/

- Xu, C., and Torres-Verdín, C. 2012, Saturation-height and invasion consistent hydraulic rock typing using multi-well conventional logs. SPWLA 53rd Annual Logging Symposium, June 16-20, 2012 Cartagena, Colombia

- Xu, C., Torres-Verdín, C. 2013. Pore System Characterization and Petrophysical Rock Classification Using a Bimodal Gaussian Density Function. Math Geosci DOI 10.1007/s11004-013-9473-2

- Yun, M., Yu, B., Xu, P., Cai, J. 2008. Fractal analysis of power-law fluid in a single capillary. Chinese Physics Letters, 25 (2), 616-619.

- Zhang, P., Lu, S., Li, J., Zhang, J., Xue, H., Chen, C. Permeability evaluation on oil-window shale based on hydraulic flow unit: A new approach. Advances in Geo-Energy Research, 2018, 2(1): 1-13, doi: 10.26804/ager.2018.01.01. 